\title{
An Overview of Booster and AGS Polarized Proton Operation During Run 15
}

\author{
K. Zeno
}

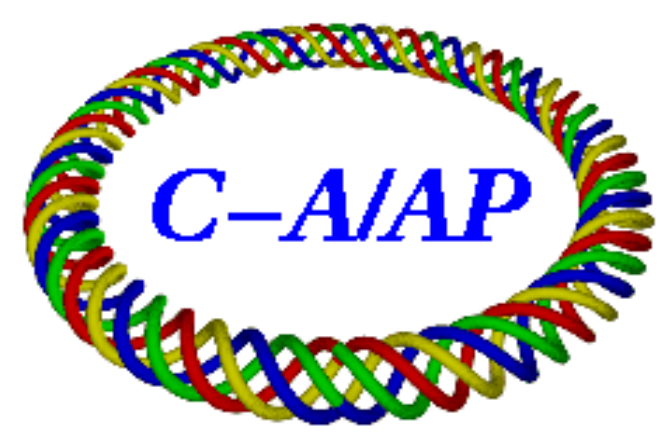

\section{Collider-Accelerator Department Brookhaven National Laboratory \\ Upton, NY 11973}

\section{U.S. Department of Energy} Office of Science, Office of Nuclear Physics

Notice: This document has been authorized by employees of Brookhaven Science Associates, LLC under Contract No. DE-SC0012704 with the U.S. Department of Energy. The United States Government retains a nonexclusive, paid-up, irrevocable, world-wide license to publish or reproduce the published form of this document, or allow others to do so, for United States Government purposes. 


\section{An Overview of Booster and AGS Polarized Proton Operation During Run 15}

Keith Zeno 
This note is an overview of the Booster and AGS for the 2015 Polarized Proton RHIC run from an operations perspective. There are some notable differences between this and previous runs. In particular, the polarized source intensity was expected to be, and was, higher this year than in previous RHIC runs. The hope was to make use of this higher input intensity by allowing the beam to be scraped down more in the Booster to provide a brighter and smaller beam for the AGS and RHIC. The RHIC intensity requirements were also higher this run than in previous runs, which caused additional challenges because the AGS polarization and emittance are normally intensity dependent.

\section{Booster $2^{\text {nd }}$ RF Harmonic}

Multiwire measurements in BtA previous to this run showed that the transverse beam size grows at these higher input intensities (>4-5e11), and if the transverse beam size grows one cannot take full advantage of the higher input to provide a brighter beam. The beam size in LtB does not appear to be intensity dependent, so the emittance growth occurs in the Booster, and is likely related to space charge forces. So this year, a $2^{\text {nd }}$ RF harmonic was used to reduce the peak charge density early in the Booster cycle where the space charge forces have the largest effect.

During Run 15, a $2^{\text {nd }}$ RF harmonic was used in the Booster for the first time since high intensity proton running. There are 2 sets of RF cavities in the Booster; A3/B3 and A6/E6. The available frequency range of A3/B3 is much greater than that of A6/E6. The main accelerating voltage had been, and still is, $\mathrm{h}=1$ and both $\mathrm{A} 3 / \mathrm{B} 3$ and A6/E6 are capable of running over the required frequency range for $h=1$ ( $\sim 0.840$ to $1.363 \mathrm{MHz}$ ). However, only A3/B3 is capable of running over the required frequency range for $h=2$. For a while now, A3/B3 have been used to accelerate the beam on $\mathrm{h}=1$. This year A6/E6 were primarily used as the $\mathrm{h}=1$ accelerating voltage. ${ }^{1}$

When the $2^{\text {nd }}$ harmonic $\mathrm{Rf}$ voltage is added to the $1^{\text {st }}$ harmonic voltage with the 180 degree point on the $2^{\text {nd }}$ harmonic centered on the synchronous phase of the $\mathrm{h}=1 \mathrm{Rf}$ wave, and the relative amplitudes of the 2 waves are set appropriately, a region of constant Rf voltage is created in the resulting Rf wave. Within this flat region, the restoring force is nearly zero and the shape of the bunch becomes flat instead of peaked. This reduces the peak charge density, which reduces the effects of space charge.

Figure 1 is a mountain range of the wall current monitor during the first $10 \mathrm{~ms}$ or so of acceleration with no $2^{\text {nd }}$ harmonic, and Figure 2 shows the same period with the $2^{\text {nd }}$ harmonic on and configured to flatten the bunches. It's clear from these 2 pictures that the bunches are flatter and also wider when the $2^{\text {nd }}$ harmonic is on. This is the configuration that was used during the run.

\footnotetext{
${ }^{1}$ They had not been used for this purpose for many years, though over the past few years they have been used for merging heavy ion beams from $\mathrm{h}=4$ to 2 .
} 
Figure 3 shows the normalized current transformer in both of these cases for an input of 8.8e11. Note that there is more early loss when the $2^{\text {nd }}$ harmonic is not on (the darker yellow trace). The

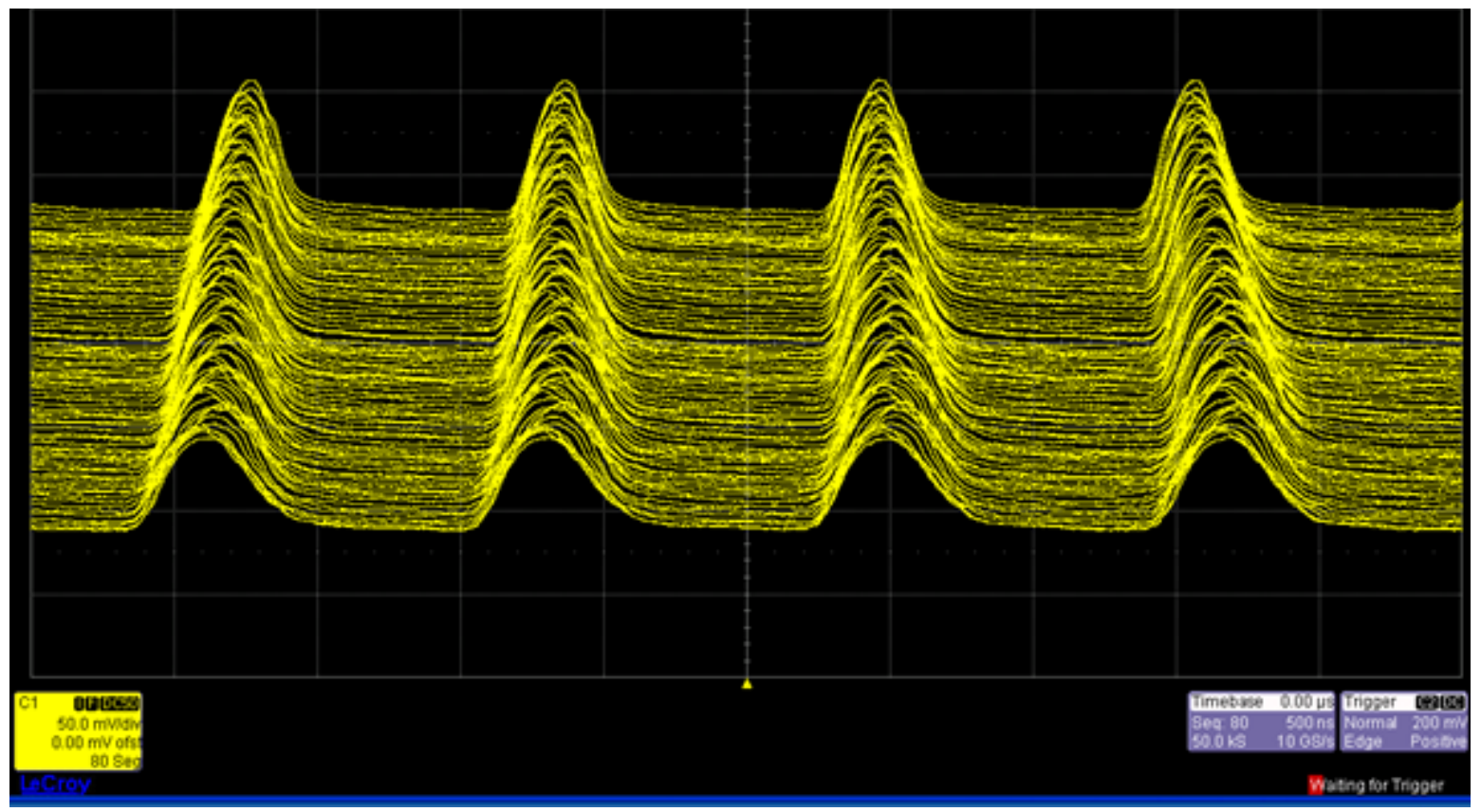

Figure 1: First 10 ms of acceleration with the $2^{\text {nd }}$ harmonic off. $^{2}$

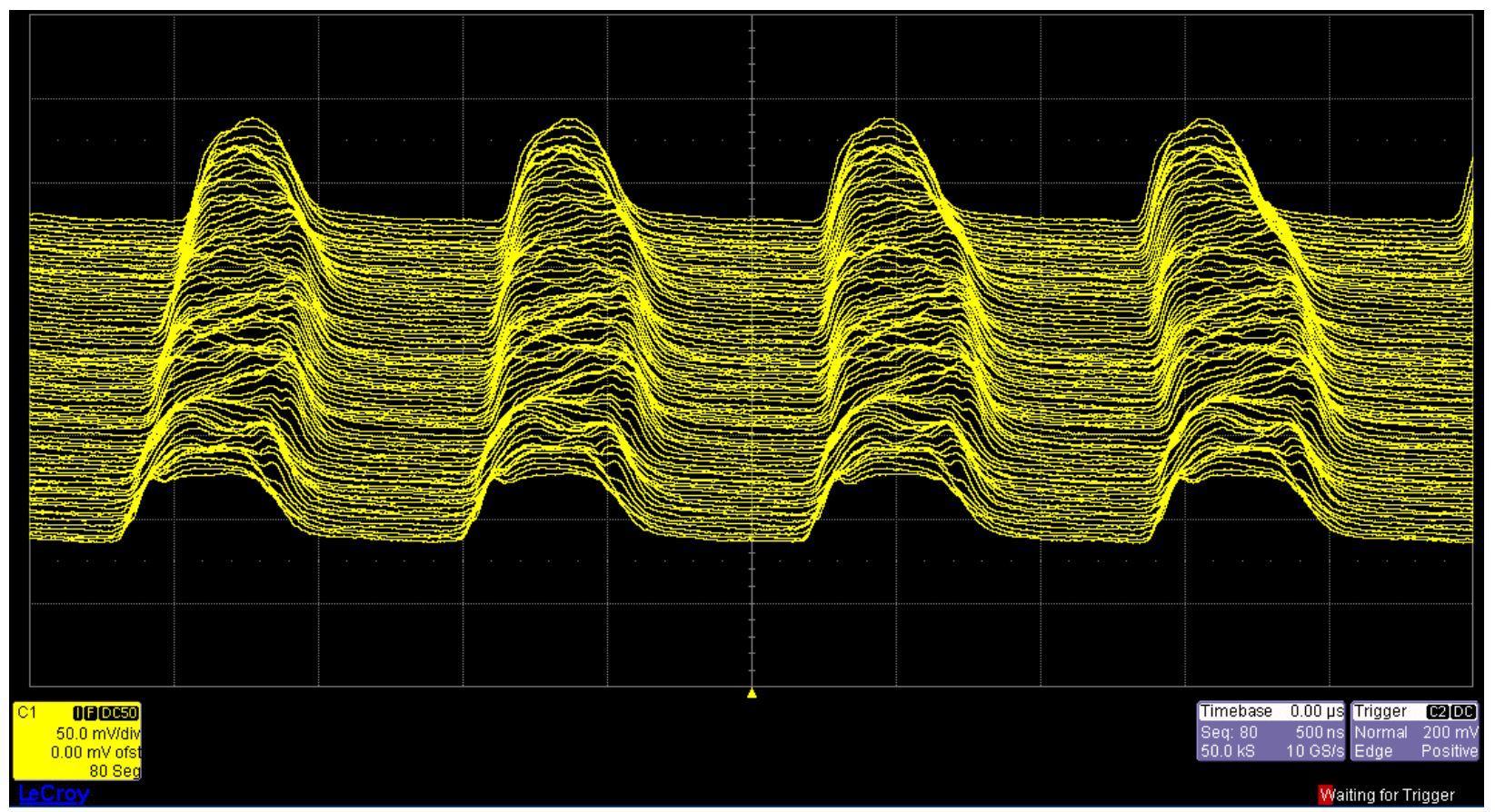

Figure 2: The first $10 \mathrm{~ms}$ of acceleration with the $2^{\text {nd }}$ harmonic on. ${ }^{3}$

\footnotetext{
2 Jan 23, 2015 17:37entry in Booster-AGS PP elog

3 Jan 23, 2015 17:38 entry in Booster-AGS PP elog
} 
large loss towards the end of the cycle is vertical scraping. In this case more beam gets through the vertical scrape when the $2^{\text {nd }}$ harmonic is on. During the early part of the cycle there are (at least) two types of loss, the first is the relatively sharp loss about $10 \mathrm{~ms}$ after injection. That is likely a loss of beam that is not captured in the RF buckets as the main magnet starts to ramp up. Presumably the bucket is larger with the $2^{\text {nd }}$ harmonic on and so more of the beam is captured. The second slower loss is intensity dependent and may be associated with losses due to stopbands (this loss is absent at lower input intensities and gets larger than this if the input is higher than this). Regardless, the $2^{\text {nd }}$ harmonic reduces the size of this loss. As can be seen from the $2^{\text {nd }}$ harmonic voltage signal (red trace), the $2^{\text {nd }}$ harmonic is only on during the first part of the cycle.

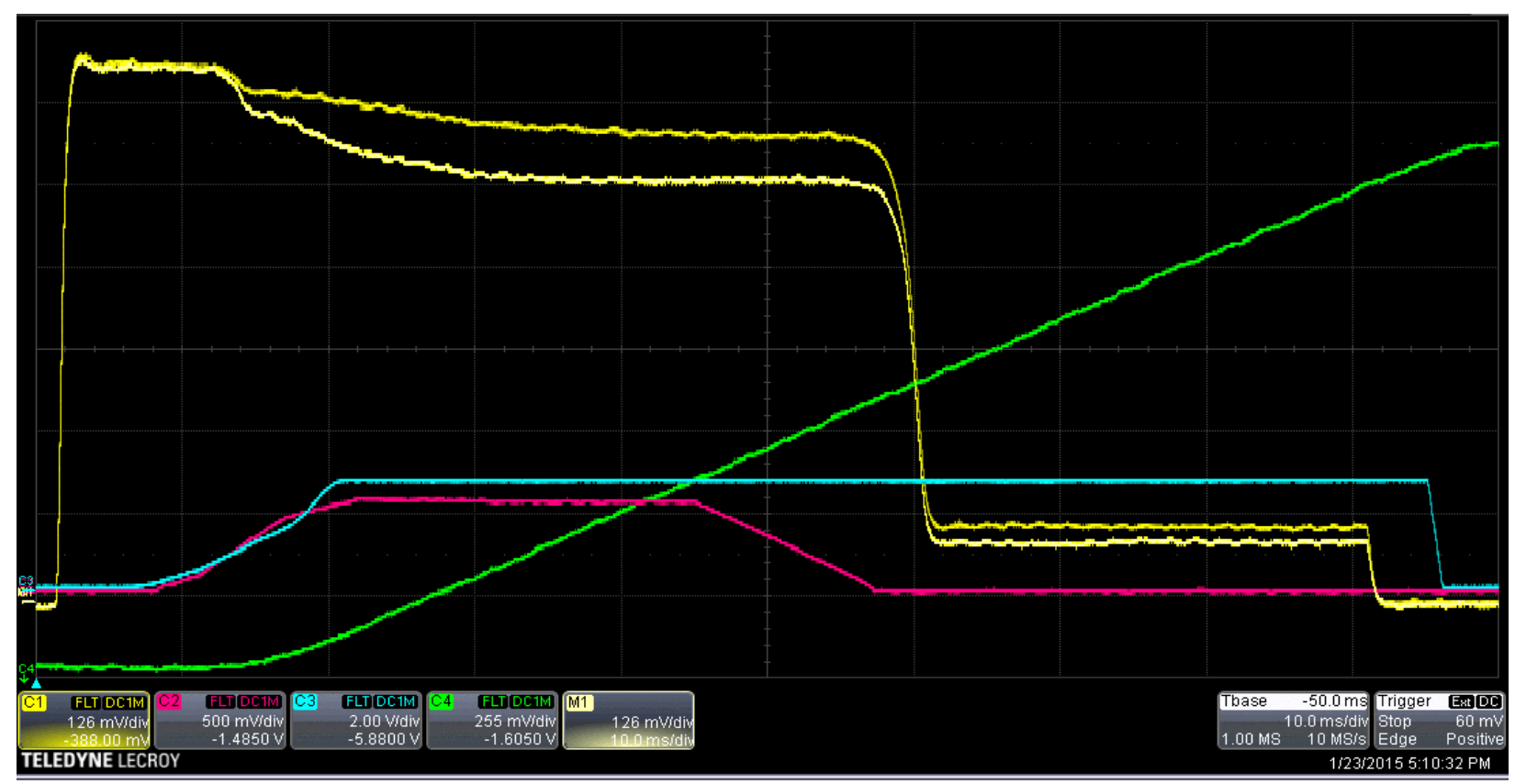

Figure 3: The Booster normalized current transformer with (C1) and without the $2^{\text {nd }}$ harmonic (M1). The blue trace (C3) is an $h=1$ cavity voltage signal, the red ( $C 2$ ) an $h=2$ cavity voltage signal (with $h=2$ is on), and the green trace is the main magnet current. The above mountain range displays were taken over a $10 \mathrm{~ms}$ interval starting around the time of the first sharper loss. Triggered at Bt0+70 ms with a sweep speed is $10 \mathrm{~ms} / \mathrm{div}^{4}$

A study of the BtA multiwire profiles for input intensities from 4 to $8 \mathrm{e} 11$ with and without the $2^{\text {nd }}$ harmonic (a.k.a. $h=2$ ) was done in January using multiwire MW006. ${ }^{5}$ The input intensity was varied by changing the OPPIS Rb cell temperature. There has been no indication that the width of the beam profile in LtB is intensity dependent, so any variations are thought to be a result of processes in the Booster.

Figure 4 shows a set of MW006 profiles of unscraped beam from the study. The horizontal profile is reasonably Gaussian, but the vertical profile has large tails. The top vertical profile is shown with a Gaussian fit of all the data, in the bottom vertical profile only the points

\footnotetext{
4 Jan 23, 2015 17:09 entry in Booster-AGS PP elog

5 Booster-AGS PP 2015 elog, Jan 92015
} 
around the core are used for the fit. One can see that the Gaussian fit for all the data is not good, but the fit of only the core is a good fit of the core. So, quantifying the vertical size is not so easy.
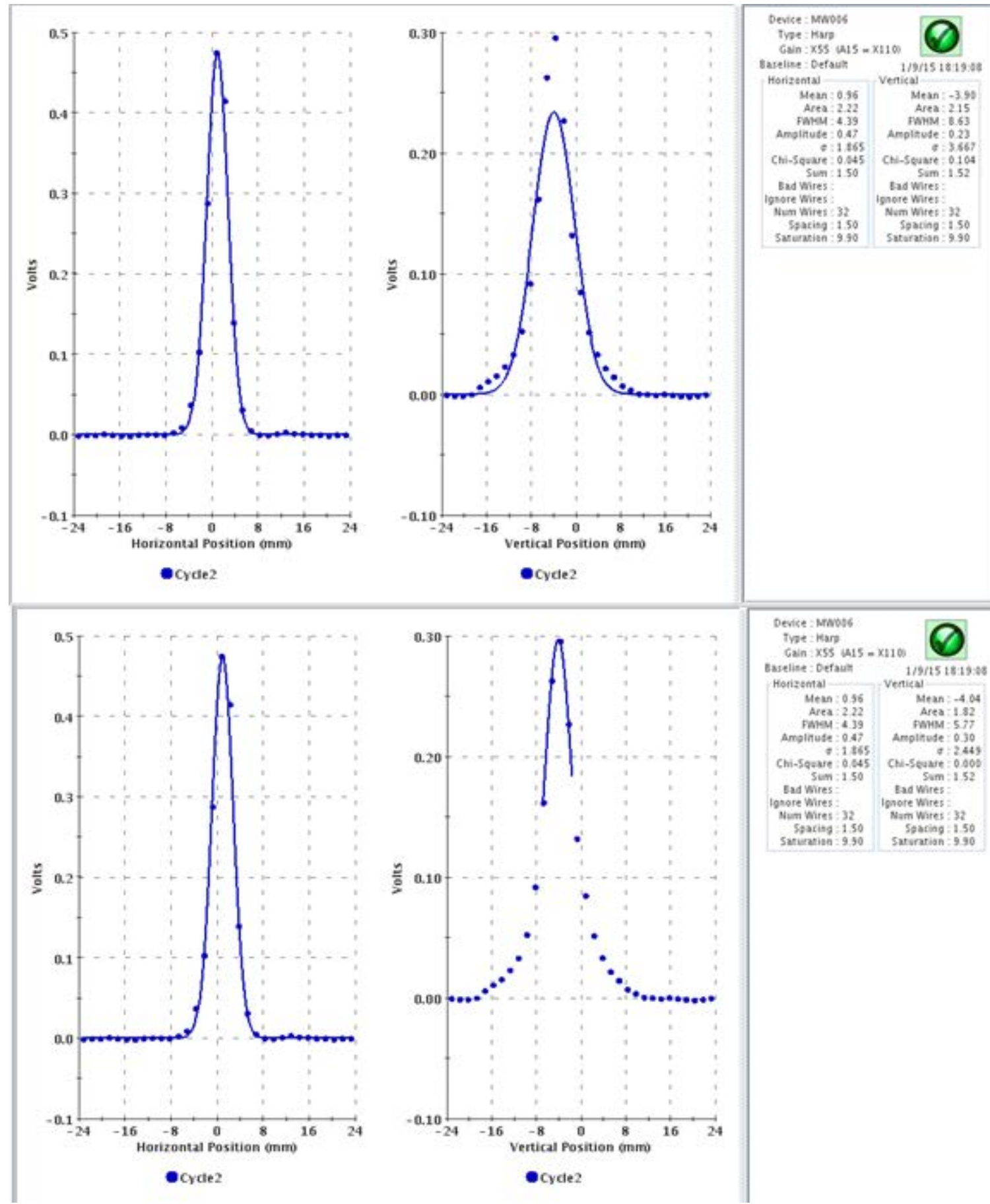

Figure 4: BtA MW006 profile data with Gaussian fits. The horizontal is on the left and vertical on the right. The top vertical profile uses all the points to obtain a Gaussian fit, the bottom one uses only the points in the core. 
Figure 5 is a plot of the FWHMs (Full Width Half Max) of the fits over a range of input intensities with both $\mathrm{h}=2$ on and off. Regardless of whether $\mathrm{h}=2$ is on or off the vertical Gaussian fits of the core and of all the data converge as the input intensity is increased. The horizontal profile doesn't show any obvious intensity dependence in either case. In the vertical, at the highest intensity the FWHMs of the fit of the core and of the entire profile are somewhat farther away from each when $\mathrm{h}=2$ is on, though the difference is not large. Hopefully, when the beam is scraped vertically it is the width of the core that's most important for providing the brightest beam. In that case, at the highest input intensity studied (8e11) the $\mathrm{h}=2$ on case is slightly smaller. But even if they were the same, the intensity of the beam extracted from the Booster is about $10 \%$ higher with $\mathrm{h}=2$ on at that input intensity.

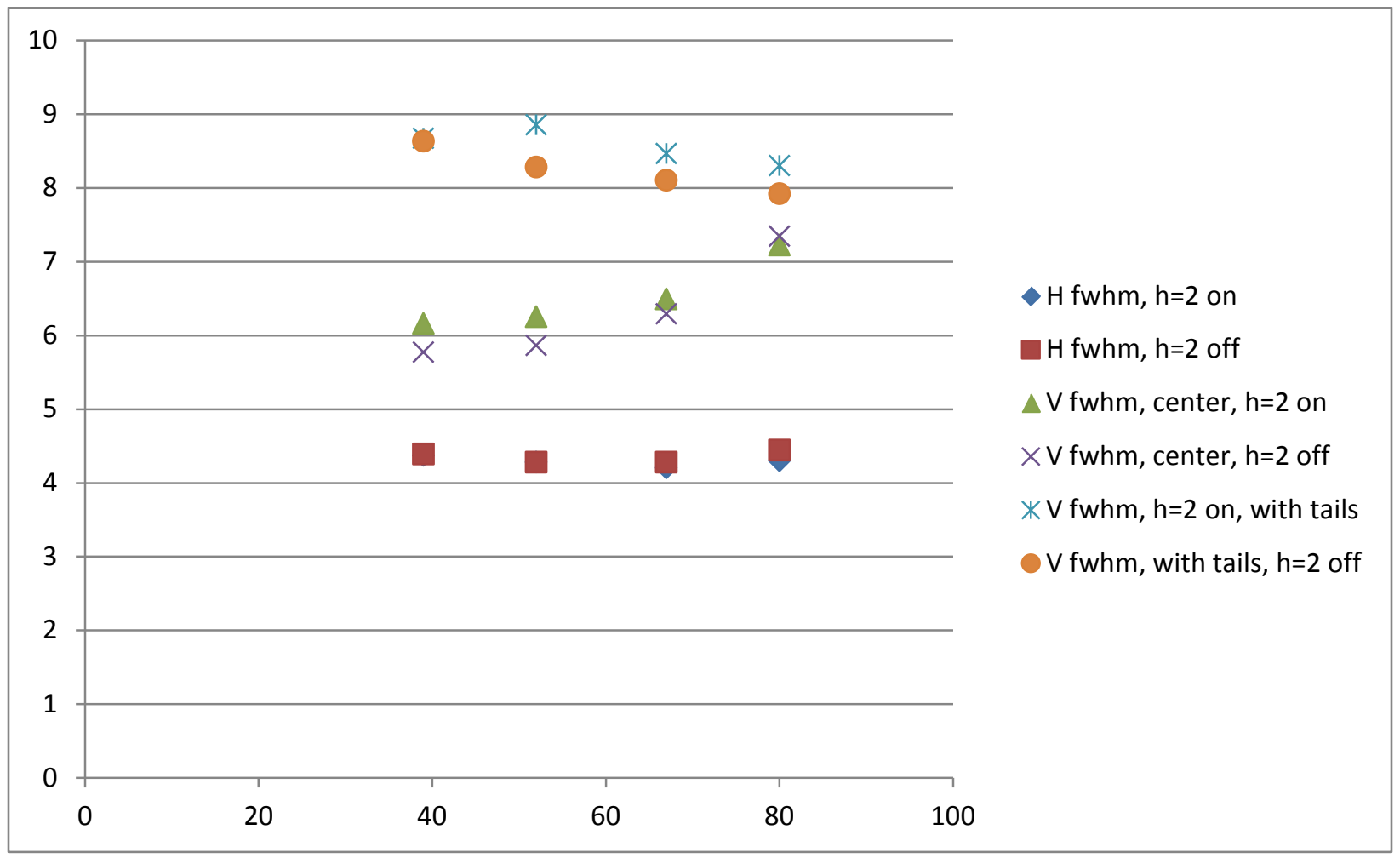

Figure 5: The FWHMs of Gaussian fits of the horizontal and vertical profiles using BtA mw006 with $\mathrm{h}=\mathbf{2}$ on and off. For the vertical both a fit of the center and of the entire profile are shown. The $x$-axis is input intensity $\left(x 10^{10}\right)$ and the $y$-axis is the FWHM (in $\mathrm{mm}$ ).

Figure 6 shows the Booster late intensity as a function of input intensity for a constant amount of vertical scraping and for no scraping at all. The lines are extrapolations from the $h=2$ on data at the lowest intensity. They are meant to show what the relationship between input and late intensity would be if there were negligible intensity dependence. Note that the $\mathrm{h}=2$ case shows some, but less intensity dependence. With an input of 8e11, which is slightly less than the nominal input intensity for the run ( 9e11), the late intensity is about $10 \%$ higher in the case without scraping and about $7 \%$ higher with this vertical scraping. 


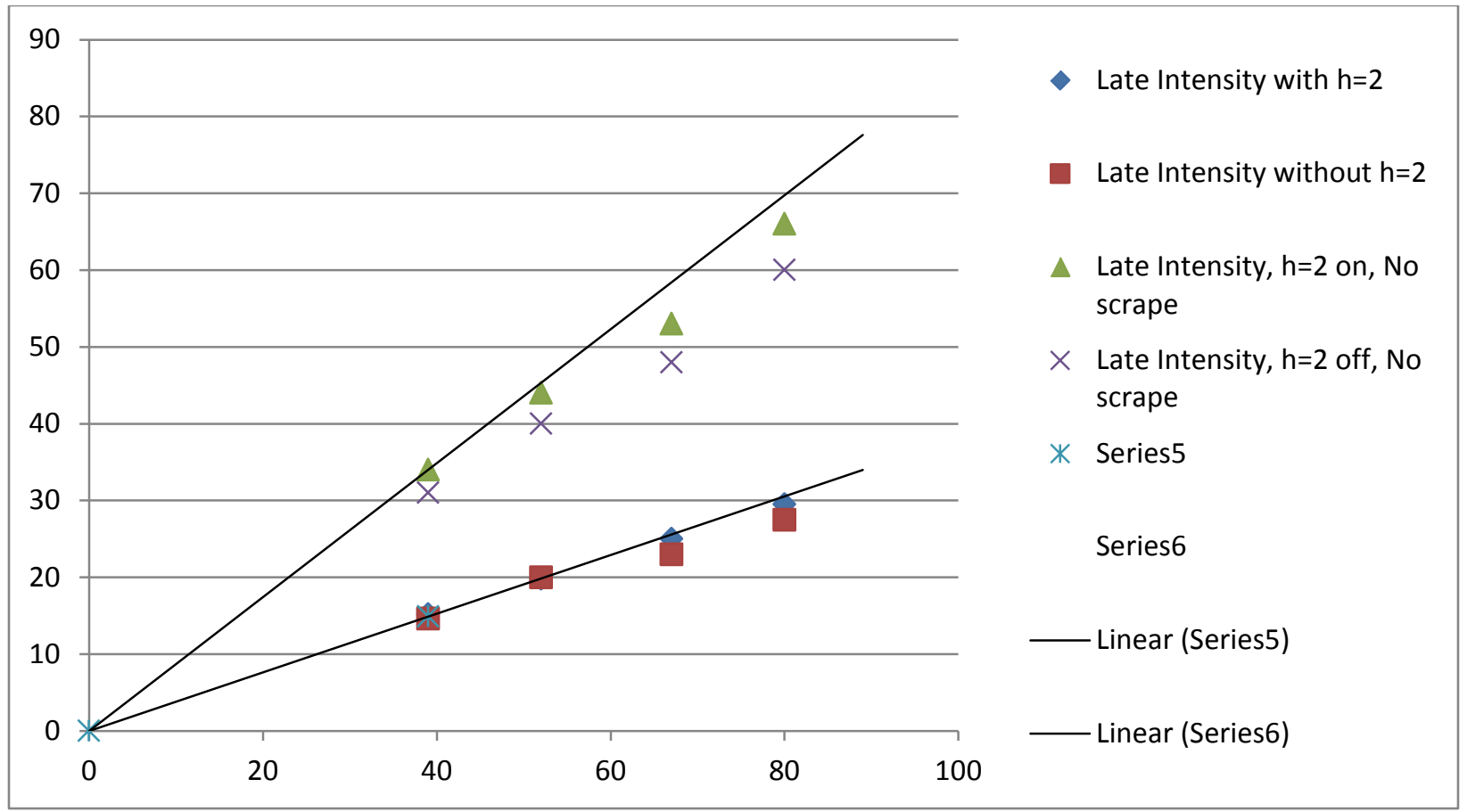

Figure 6: Input vs Booster late intensity with $\mathrm{h}=\mathbf{2}$ on and off for the case of no scraping and a fixed amount of vertical scraping. The lines are extrapolated from the data at the lowest input intensity (3.9e11) and are intended to represent what the relationship between the two would be like without intensity dependence. The $x$-axis is the Booster input intensity $\left(x 10^{10}\right)$, and the $y$-axis is the Booster late intensity $\left(\times 10^{10}\right)$.

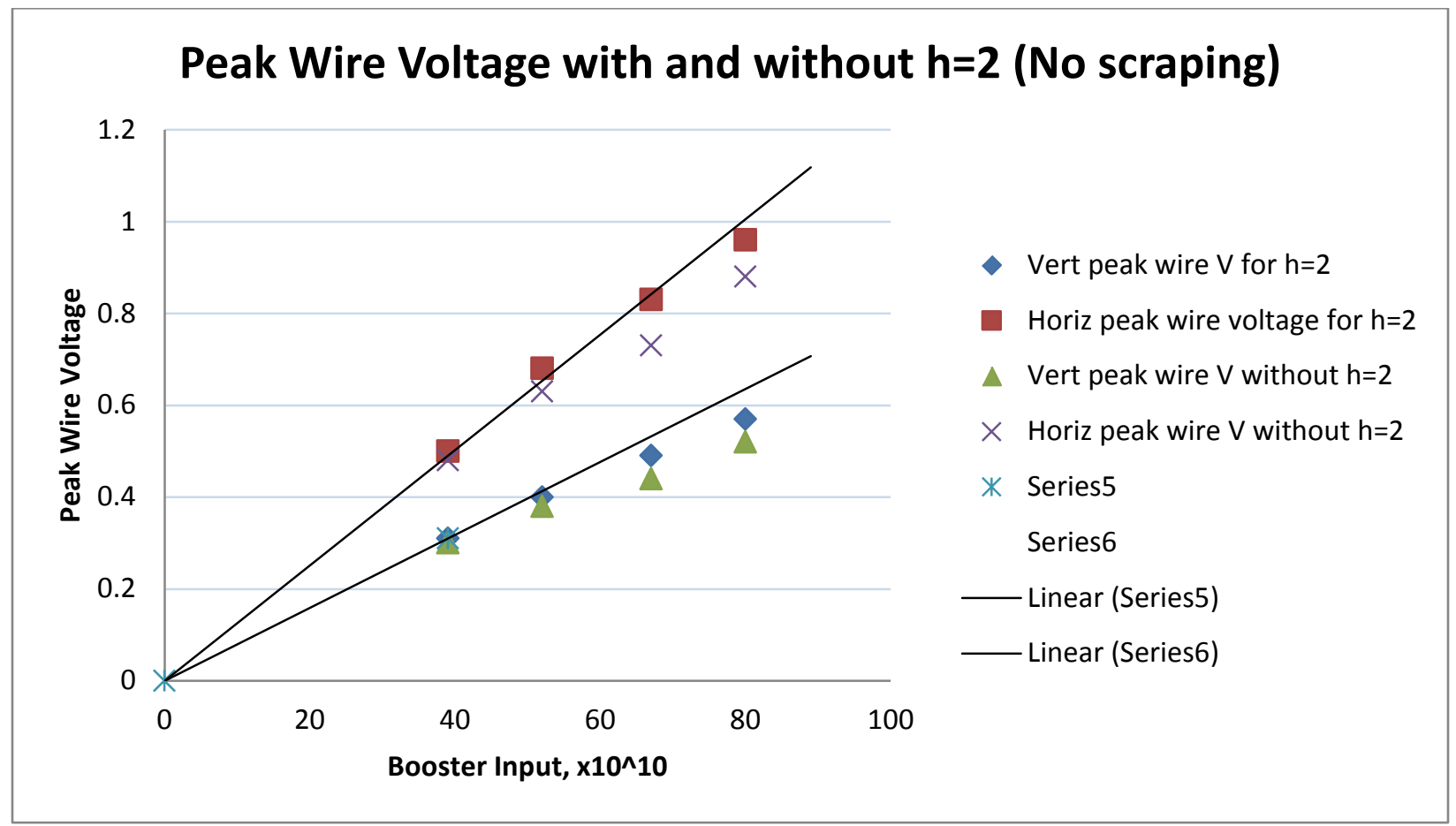

Figure 7: BtA mw006 horizontal and vertical peak wire voltages for the case of no scraping with and with the $2^{\text {nd }}$ harmonic as a function of input intensity. The lines are extrapolated from the data at the lowest input intensity (3.9e11) and are intended to represent what the relationship between the two would be like without intensity dependence. 
Figure 7 is a plot of the peak horizontal and vertical wire voltages, which are proportional to the peak charge densities, and are used to quantify the peak brightness. Once again the lines are extrapolated from the $\mathrm{h}=2$ data at lowest intensity and are meant to show what the relationship would be if there were no intensity dependence. Note that in both planes the $\mathrm{h}=2$ case shows less intensity dependence. In the horizontal there is not an obvious dependence for the $h=2$ on case, but there is some for the $h=2$ off case. In the vertical there is some dependence for both, but less when the $h=2$ is on.

\section{New Booster Main Magnet Cycle}

In hopes of further reducing any intensity dependent effects, a new Booster main magnet cycle with a faster early acceleration ramp was used for most of the run. This change took effect on Feb 24. Figure 8 shows the former and the new magnet cycle. Beam is injected at Bt $0+71.2$ ms which is about $10 \mathrm{~ms}$ before the main magnet starts to ramp. In the new cycle the main magnet reaches the peak dB/dt several ms earlier and the peak dB/dt is higher (65 vs. $49 \mathrm{~g} / \mathrm{ms}$ ). Injection occurs at the same time in the cycle, and extraction happens $1 / 60^{\text {th }}$ of a second earlier (Bt0+144.2 vs. $160.9 \mathrm{~ms}$ ). Extraction also happens at a lower dB/dt (28 vs. $49 \mathrm{~g} / \mathrm{ms}$ ) which allows for a lower RF voltage at extraction. This lower voltage allows wider bunches to be injected into the AGS for the same longitudinal emittance, which might help a little if there are any space charge effects near injection in the AGS.

It's not clear whether the faster cycle helped improve the transverse brightness of the beam. If it did, the effect is small at the intensities that were run this year. Also, A6/E6 can provide significantly more voltage than A3/B3. Using the faster cycle would not have been possible if only A3 and B3 were used for the accelerating voltage as in previous years.

\section{Vertical Tune Early in the Booster Cycle}

Additionally, raising the vertical tune during the early part of the cycle helps with beam survival and transverse brightness. This was the case years ago during high intensity proton operation, so it further supports the idea that space charge forces are responsible for the intensity dependence. The space charge forces not only depress the individual particle tunes, but spread out the tune distribution. Raising the vertical tune close to the integer may help with moving that distribution out of the range of the third integer resonances. Figure 9 shows the set vertical tune through the early part of the cycle for this year and run 13. This setting is tuned to optimize intensity and beam size in BtA. This year its optimal setting was considerably higher than in Run 13.

At an input of 10.7e11, about 20\% higher than normal running, the third integer (normal) stopband corrections have been shown to have a significant effect on the amount of beam that passes through both the horizontal and vertical scrapes. ${ }^{6}$ This suggests that at least at that

${ }^{6}$ Feb $6{ }^{\text {th }}$ Booster-AGS PP elog 1522 and 1535 entries 
intensity, individual particle tunes are within the range of those stopbands which means that the actual particles tunes are far from the set tunes.

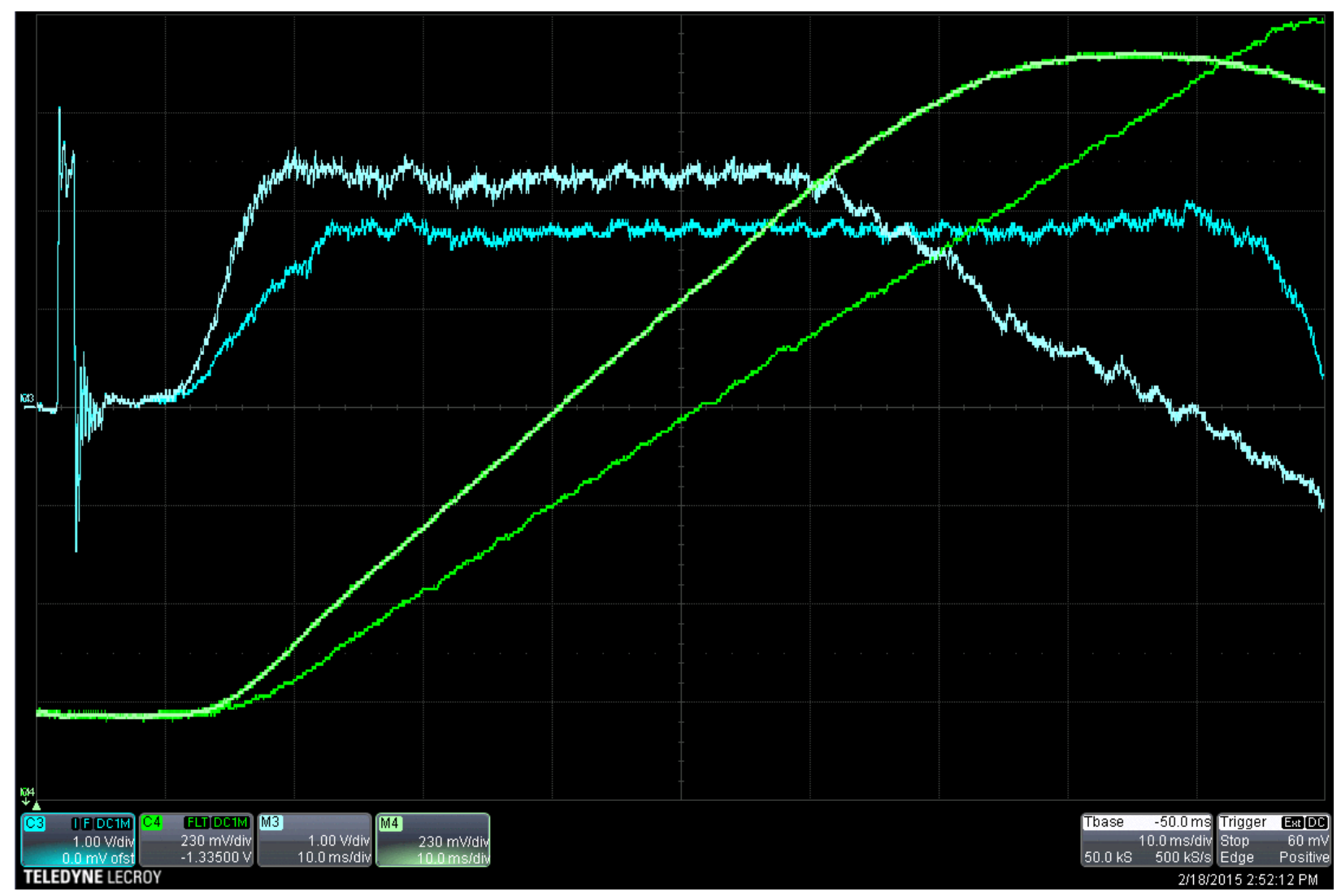

Figure 8: The former and the new Booster main magnet cycle. The blue traces are a dB/dt signal for both magnet cycles; the green traces are the main magnet current for both cases. The lighter colored traces are for the new magnet cycle, the darker for the old one. The trigger is Bt0+70 ms and the sweep speed is $10 \mathrm{~ms} / \mathrm{div}$.

Table I shows a series of vertical tune measurements that were made during the early part of the cycle for inputs of $1.5 \mathrm{e} 11$ and $7.8 \mathrm{e} 11$ as well as the difference between them $(\Delta \mathrm{Qv})$. There is a small but measurable depression in the measured $\mathrm{Qv}$ between the lower and higher intensities. The depression is largest from 80 to $95 \mathrm{~ms}$. There was no difference apparent in $\mathrm{Q}_{\mathrm{h}}$. $\mathrm{Qv}$ was also measured for the 7.8e11 case with and without $\mathrm{h}=2$. No difference was seen at 80 and $95 \mathrm{~ms}$, and only a very small difference was seen at $90 \mathrm{~ms}(\Delta \mathrm{Qv}=-0.001)$. It remains a question whether this small difference in the coherent $\mathrm{Qv}$ is consistent with an incoherent tune spread large enough to cause intensity dependent problems.

As to the question of whether the set tunes follow the measured tunes, measurements were made on Feb $3^{\text {rd }}$ throughout the cycle and compared to the set tunes. Figure 10 shows the results which indicate fairly good agreement. The average radius through the cycle was to the outside, which could explain at least some of the discrepancy. This is especially so in the horizontal since the horizontal chromaticity is significant and negative and the measured tune is lower than the set tune. 


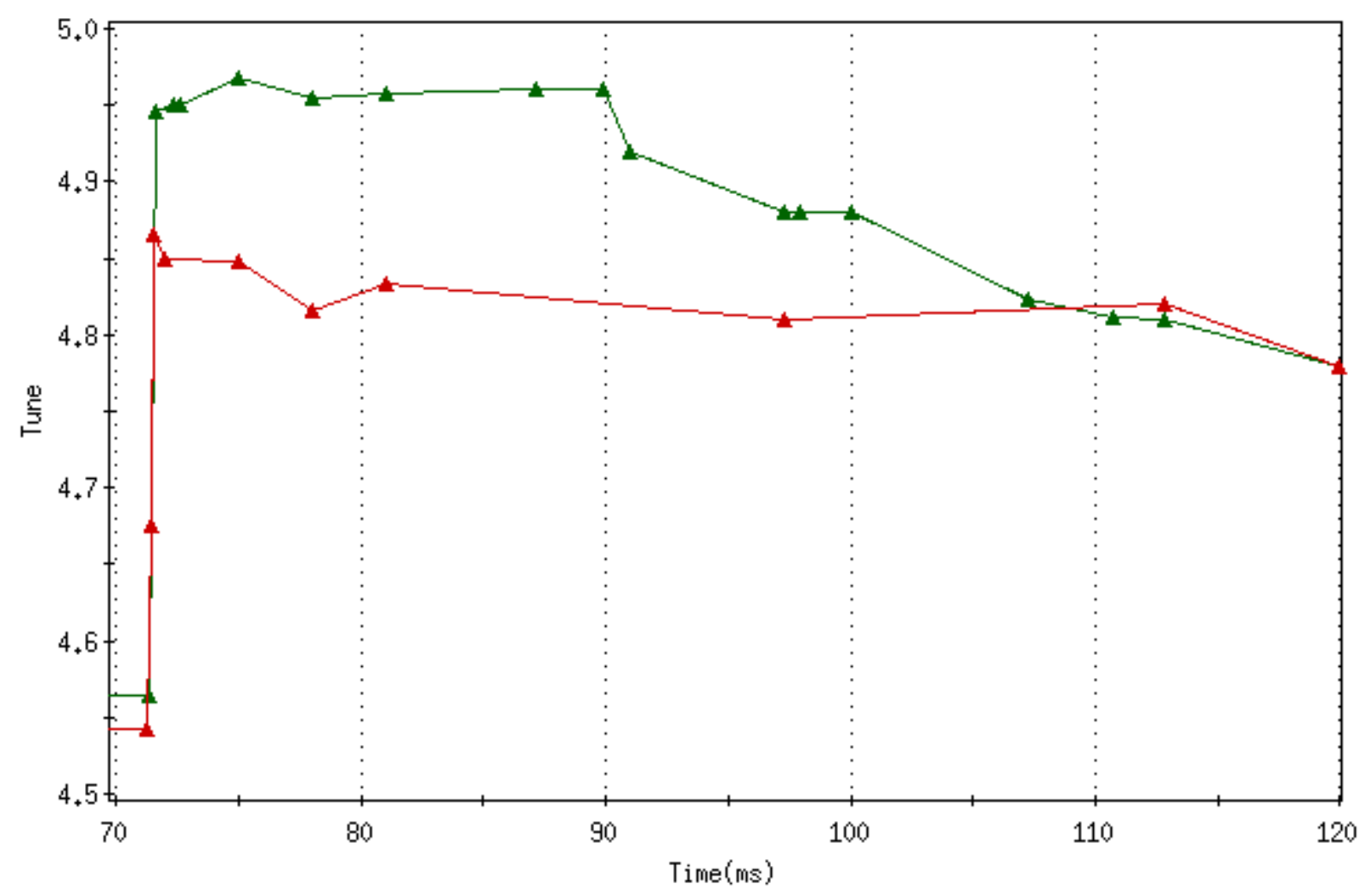

$\longrightarrow$ V_tune_desired(t)/Mon\#02231840_Boo_4 $\longrightarrow$ V_tune_desired_t_/Tue\#04150636_Bo0_4

Figure 9: Typical Booster vertical tune settings for Run 15 (green) and Run 13 (red). The x-axis is time from Bt0 in ms. The Run 15 settings are from an archive before the magnet cycle change was made.

\begin{tabular}{|c|l|c|c|c|c|c|}
\hline Intensity & & $\mathbf{8 0 m s}$ & $\mathbf{8 5 m s}$ & $\mathbf{9 5 m s}$ & $\mathbf{1 0 5 m s}$ & $\mathbf{1 2 0 m s}$ \\
\hline $1.5 \mathrm{e} 11$ & $\mathrm{Q} v$ & 4.922 & 4.884 & 4.857 & 4.803 & 4.763 \\
\hline $7.8 \mathrm{e} 11$ & $\mathrm{Q} v$ & 4.917 & 4.879 & 4.852 & 4.801 & 4.762 \\
& & & & & & \\
\hline & $\Delta \mathrm{Qv}$ & -0.005 & -0.005 & -0.005 & -0.002 & -0.001 \\
\hline
\end{tabular}

Table I: Measurements of Qv in the Booster for 2 different input intensities. Times are from Bt0. These measurements were done with the slower main magnet cycle that was used before Feb $24 .^{7}$

\footnotetext{
${ }^{7}$ Jan 23 Booster-AGS PP elog, most entries between 1432 and 1525.
} 


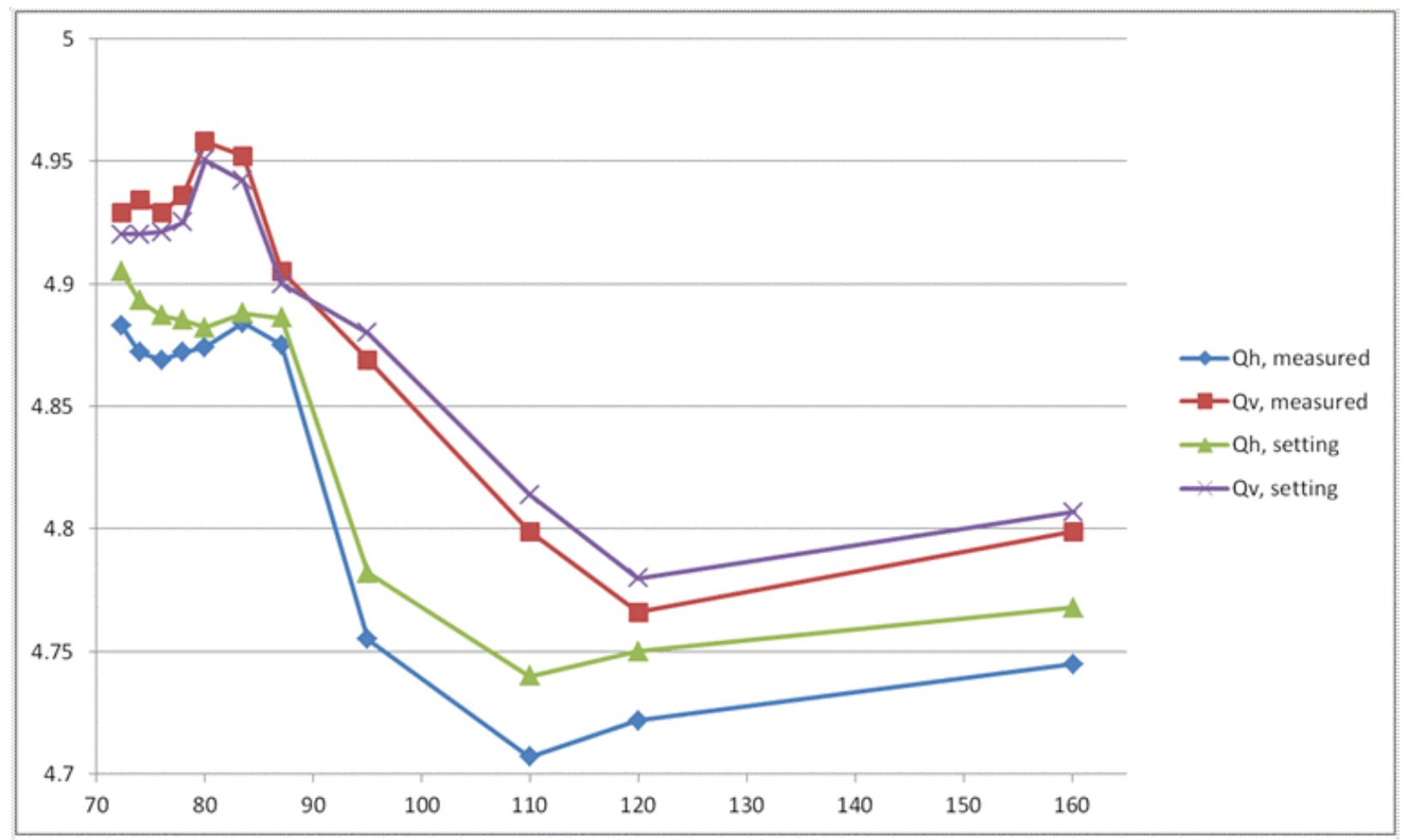

Figure 10: Measured vs. set tunes vs. time in the Booster cycle. This is with the slower magnet cycle and normal (not half integer) injection tunes. The $x$-axis is time in $\mathrm{ms}$ from Bt0. ${ }^{8}$

\section{Booster Injection}

The half integer injection scheme, which has been used since 2009, was used for the vast majority of the run. The scheme consists of setting both betatron tunes just above the half integer (4.5 in the Booster) and adjusting the half-integer stopband correctors to minimize the transverse emittance (measured by beam size in BtA and/or how much intensity gets through a fixed amount of scraping later in the cycle). When the tunes are close to the half integer, the half integer stopband correctors distort the $\beta$ functions at the $\mathrm{H}$ - stripping foil. It has been demonstrated that adjusting the half integer stopband correctors at injection can reduce the effect that scattering on the foil has on the transverse emittance. ${ }^{9}$

Due to the proximity of the injection tunes to the half-integer, and the large currents in the half integer stopband correctors (which are there to distort the beta functions, not correct the half integer stopband), the tunes are moved as quickly as possible away from the half integer after injection is complete. This can be seen in Figure 9, where injection occurs around 71.2-71.5 $\mathrm{ms}$ from Bt0. Additionally, the Rf voltage is set to zero until the end of the injection process, then it is raised slowly from zero so that the tunes are away from the half integer before there is

\footnotetext{
${ }^{8}$ Feb 3 Booster-AGS PP elog. This data was taken with the 'normal' injection tunes scheme discussed below. It shouldn't matter though since the data is just meant to compare the set and measured tunes.

${ }^{9}$ See C-AD note, "Booster and AGS transverse emittance during the 2006 and 2009 polarized proton runs", K. Zeno, 2010 for more information on half integer injection.
} 
significant bunching. This is done because bunching would greatly increase the peak charge density and thereby potentially increase space charge's effect on the tune distribution.

Figure 11 shows the vertical tune quad current together with the Booster wall current monitor and the current in one of the injection bump magnets. The Linac pulse is injected during the last $300 \mu$ s before the injection bump starts to collapse. Just as the injection bump starts to collapse both tunes are shifted. In this case, the total shift in the vertical is about 0.43 , and in the horizontal it is about 0.25 . This shift occurs over less than a $1 \mathrm{~ms}$ interval. Note that there is very little bunching before the tunes are far from the half integer. ${ }^{10}$

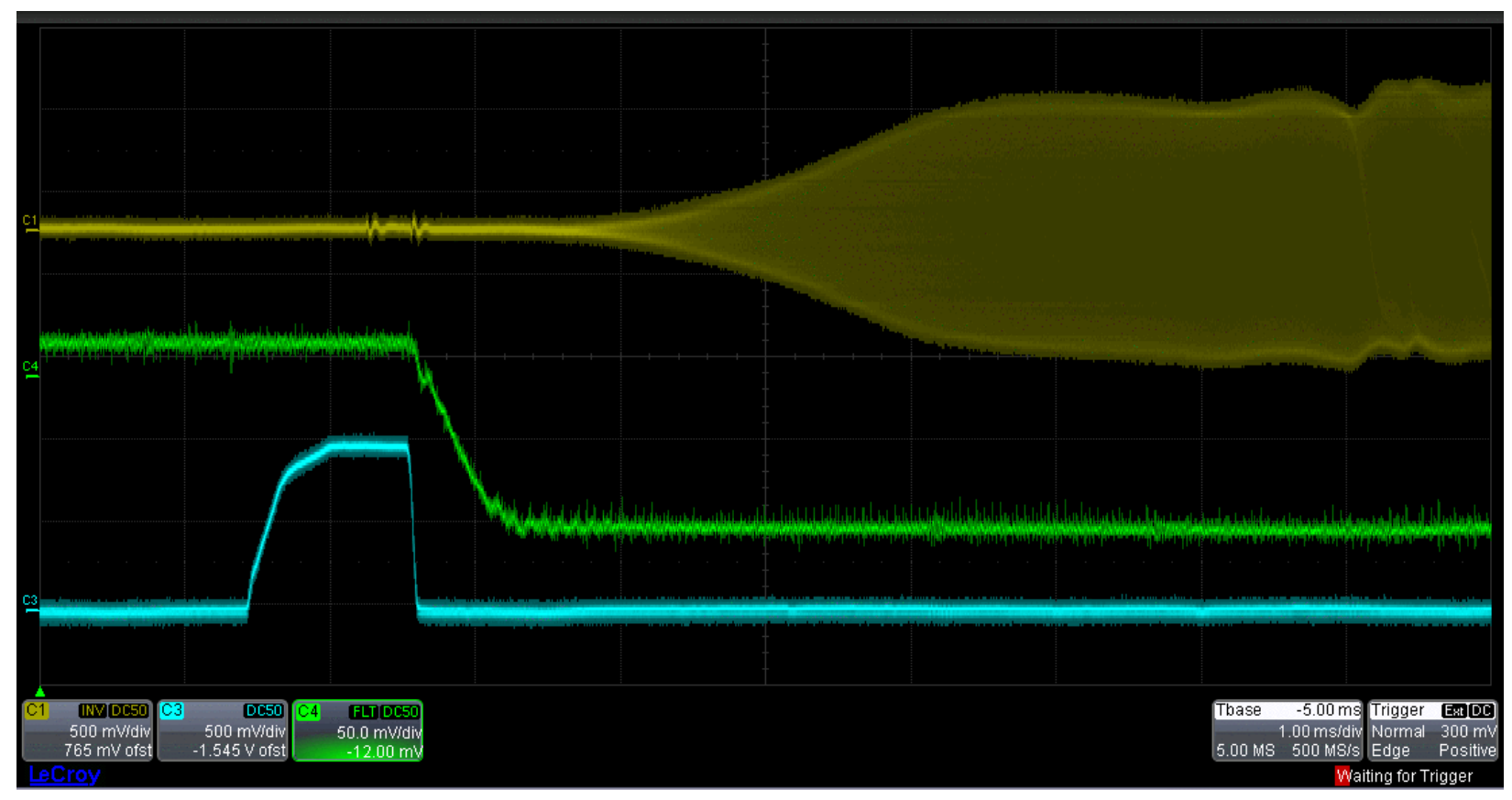

Figure 11: Booster injection and Rf capture showing the Booster wall current monitor (yellow, C1), vertical tune quad current (green, C4), and the C3 injection bump magnet current (blue, C3). Glitches in the wall current monitor at the beginning and end of the Linac pulse indicate when injection occurs. Sweep speed is $1 \mathrm{~ms} / \mathrm{div}$.

Despite these measures there was still a concern that the proximity of the higher intensity beam to the half integer resonance could cause emittance growth (or beam loss) and that this would offset any gain from this scheme. So for a period of about 6 days around the beginning of February (Jan 30-Feb 4) a 'normal' injection tune scheme was used.

A study was done on Jan 30 to compare emittance with the half integer injection scheme to a 'normal' setup with higher injection tunes. It is quite difficult to quantify the difference though because in general the optimal tune paths, in both cases, are a function of input intensity. The input intensity was varied over the range from 5.8e11 to $11.4 \mathrm{e} 11$ and the data was compared with the scan done earlier with half integer injection. Unfortunately, this range of inputs is quite

\footnotetext{
${ }^{10}$ This is actually from last year's AGS PP run, but it is very similar to the setup this year. Booster-AGS PP elog Apr 292014 at 13:55 entry
} 
different than the range covered in the half integer scan. Figure 12 compares the MW006 FWHMs for both cases ( $\mathrm{h}=2$ is on in both cases). In an attempt to cover a greater range in the half integer case I've included data taken later in the run. ${ }^{11}$

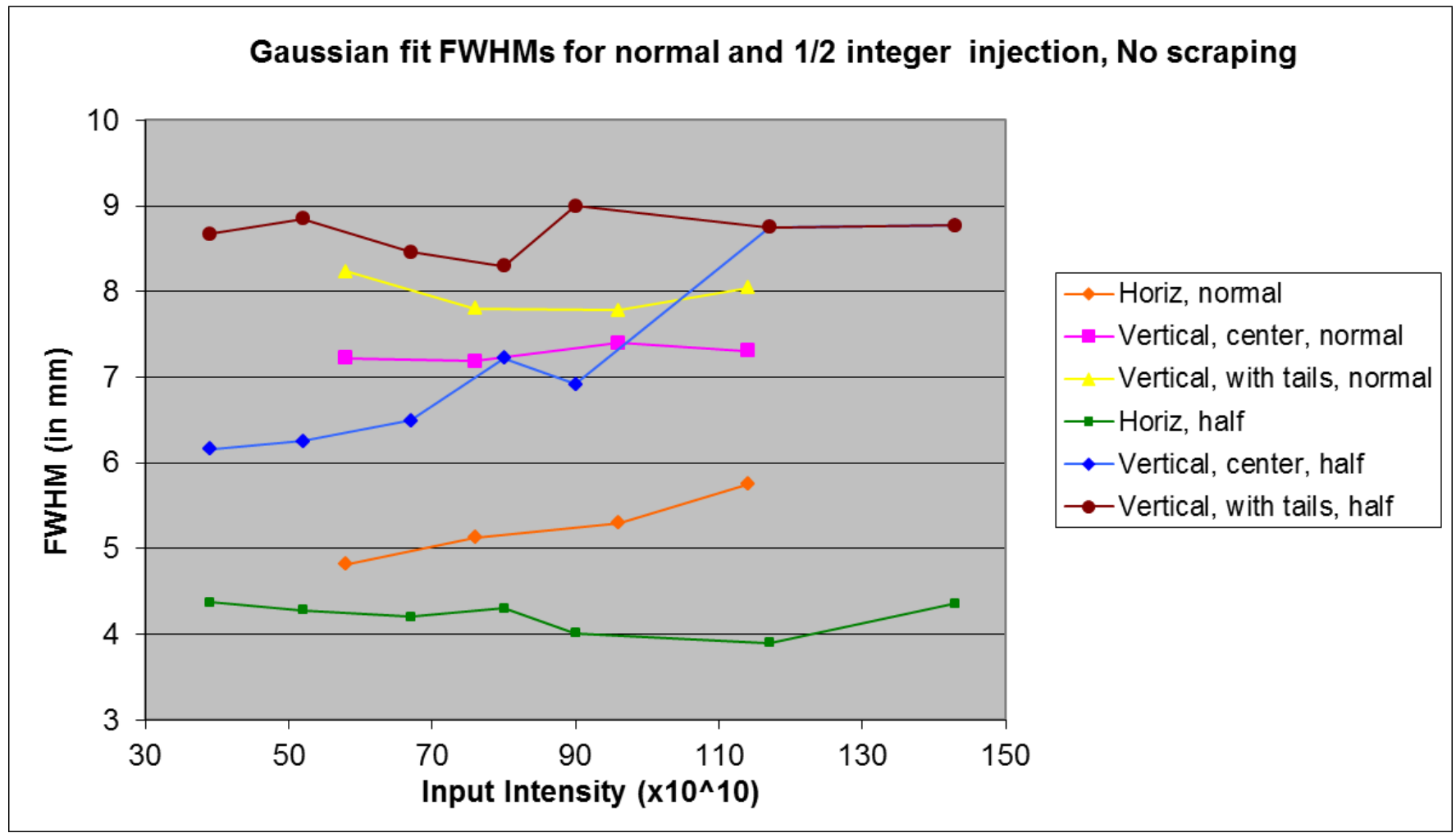

Figure 12: BtA MW006 FWHM data for the 'normal' injection scheme and half integer injection scheme. The data shown for the half integer case are the same data as the " $\mathrm{h}=\mathbf{2}$ on" case in figure 5 up to an input intensity of 8.0e11. For higher input intensities the half integer data was taken later in the run. All the 'normal' injection data was taken on Jan 30.

There are several notable things about the data. First, the horizontal width is larger and shows intensity dependence in the normal injection case but does not show intensity dependence in the half integer case. Second, the vertical is smaller and does not show intensity dependence in the normal injection case. Also, the difference between the fit of all data and the core is more pronounced in the half integer case up to an intensity of about 9.0e11, which is roughly the nominal input intensity. ${ }^{12}$

\footnotetext{
${ }^{11}$ The half integer data for Jan 9 is the $h-=2$ data shown in Figure 5. The half integer data for $9.0 e 11$ was taken on Jun 22 (with the faster magnet cycle), and the half integer data for $11.7 \mathrm{e} 11$ and $14.3 \mathrm{e} 11$ was taken on Feb 13 (slower magnet cycle). This data can be found in the Booster-AGS PP elog for those dates.

${ }^{12}$ One reason the nominal input intensity was not raised higher was because there was some drop in the source polarization apparent.
} 
For an input of 9.0e11, and using the standard values for the $\beta$ function at MW006, the average 95\% normalized emittance there $\sqrt{\varepsilon_{h}^{2}+\varepsilon_{v}^{2}} / \sqrt{2}$ is about $17 \pi \mathrm{mm}$ mrad in the normal case $\mathrm{e}^{13}$ and $13 \pi \mathrm{mm}$ mrad in the half integer case. ${ }^{14}$ This is using the vertical fit of all the data. The difference is even greater if the fits of the cores are used. So, for the nominal input intensity these intensities scans, as limited as they are, suggest that the half integer injection is preferable.

During the Run it was observed that gradually raising the vertical tune at injection in small increments as the input intensity is increased removes some of the intensity dependence. This was not done during the scans performed on Jan 9 and Jan 30, but was likely done for the data taken later in the run. ${ }^{15}$

\section{Booster Scraping and BtA}

From figure 12, a nominal unscraped horizontal fwhm on MW006 is about $4 \mathrm{~mm}$. Using $\varepsilon_{\mathrm{x}}=0.82 * \mathrm{fwhm}^{2}$, this corresponds to about $13 \pi \mathrm{mm}$ mrad. The vertical is harder to quantify. If the wires that show a beam signal are counted to determine the vertical width, a very large value is obtained for the vertical emittance because of the large tails. For example, the full width of the profile in figure 4 is 19 wires or $28.5 \mathrm{~mm}$. Using $\beta \gamma=2.3$ and $\beta_{\mathrm{y}}=16.0 \mathrm{~m}$ gives $\varepsilon_{\mathrm{y}}=29 \pi \mathrm{mm} \mathrm{mrad}$, but if the fit of the core is used it is only $5.2 \pi \mathrm{mm}$ mrad.

Figure 13 shows typical profiles for MW006 when the beam is scraped. Here the AGS late intensity is $2.5 \mathrm{e} 11$ and the input is 9.0e11. When the beam is scraped, the tails are removed so the beam is not Gaussian. Note the difference in the vertical between this and the unscraped case (Figure 4). Also note that the horizontal profile is quite narrow. Yet, despite the fact that the tails have been scraped off the Gaussian fits do not look that bad (at least sometimes, as in this case).

During a good part of the run there was a problem with MW006 and MW060 had to be used instead. ${ }^{16}$ Six measurements were made at different times during the run using both multiwires to get a calibration for MW060 (the BtA quad settings upstream of MW060 only changed by a very small amount during the run). The profiles couldn't be taken on the same cycle because the MW060 profiles are affected by MW006, but they were taken in quick succession.

\footnotetext{
${ }^{13}$ Since there isn't a data point at 9.0e11 for the normal injection case, the fwhm values were approximated using the values on the line that connects the data points.

${ }^{14} \beta x=3.0 \mathrm{~m}$ and $\beta y=16.0 \mathrm{~m}, \beta \gamma=2.3$, The ratio of $95 \%$ full width to the fwhm is 2.08 . So, $\varepsilon_{\mathrm{h}}=0.82 * \mathrm{fwhm}^{2}$ and $\varepsilon_{\mathrm{v}}=0.155 * \mathrm{fwhm}^{2}$. At $9.0 \mathrm{e} 11$ input $\varepsilon_{\mathrm{h}}=22.6$ and $\varepsilon_{\mathrm{v}}=9.4 \pi \mathrm{mm}$ mrad for the normal case, and $\varepsilon_{\mathrm{h}}=13.5$ and $\varepsilon_{\mathrm{v}}=12.6 \pi$ $\mathrm{mm}$ mrad for the half integer case. ${ }^{15}$ Feb 6 Booster-AGS PP elog 1522 entry

${ }^{16}$ Also, for a period the MW060 profiles were acquired with MW006 inserted as well. This caused the profiles on MW060 to be larger, and so that data is not accurate and so is not included in Figure 14.
} 
The equations relating the emittances to FWHMs for MW006 are $\varepsilon_{\mathrm{h}}=0.82 * \mathrm{fwhm}^{2}$ and $\varepsilon_{\mathrm{v}}=0.155 * \mathrm{fwhm}^{2}$ (see footnote 13 ). $\varepsilon_{\mathrm{h}}=0.82 * \mathrm{fwhm}_{006}{ }^{2}$ at MW006 equals $\varepsilon_{\mathrm{h}}=\mathrm{k}^{*} \mathrm{fwhm}_{060}{ }^{2}$ at MW060. So, $0.82 *$ fwhm $_{006}{ }^{2}=\mathrm{k}^{*} \mathrm{fwhm}_{060}{ }^{2}$, and $\mathrm{k}$ can be solved for. $\mathrm{k}$ was found for all 6 measurements and its average value was taken as the calibration. The same was done in the vertical. The values arrived at were $\mathrm{k}_{\mathrm{horiz}}=0.0609$ and $\mathrm{k}_{\mathrm{vert}}=0.0855$. Figure 14 shows a comparison of the measurements using those calibrations. The agreement is pretty good.
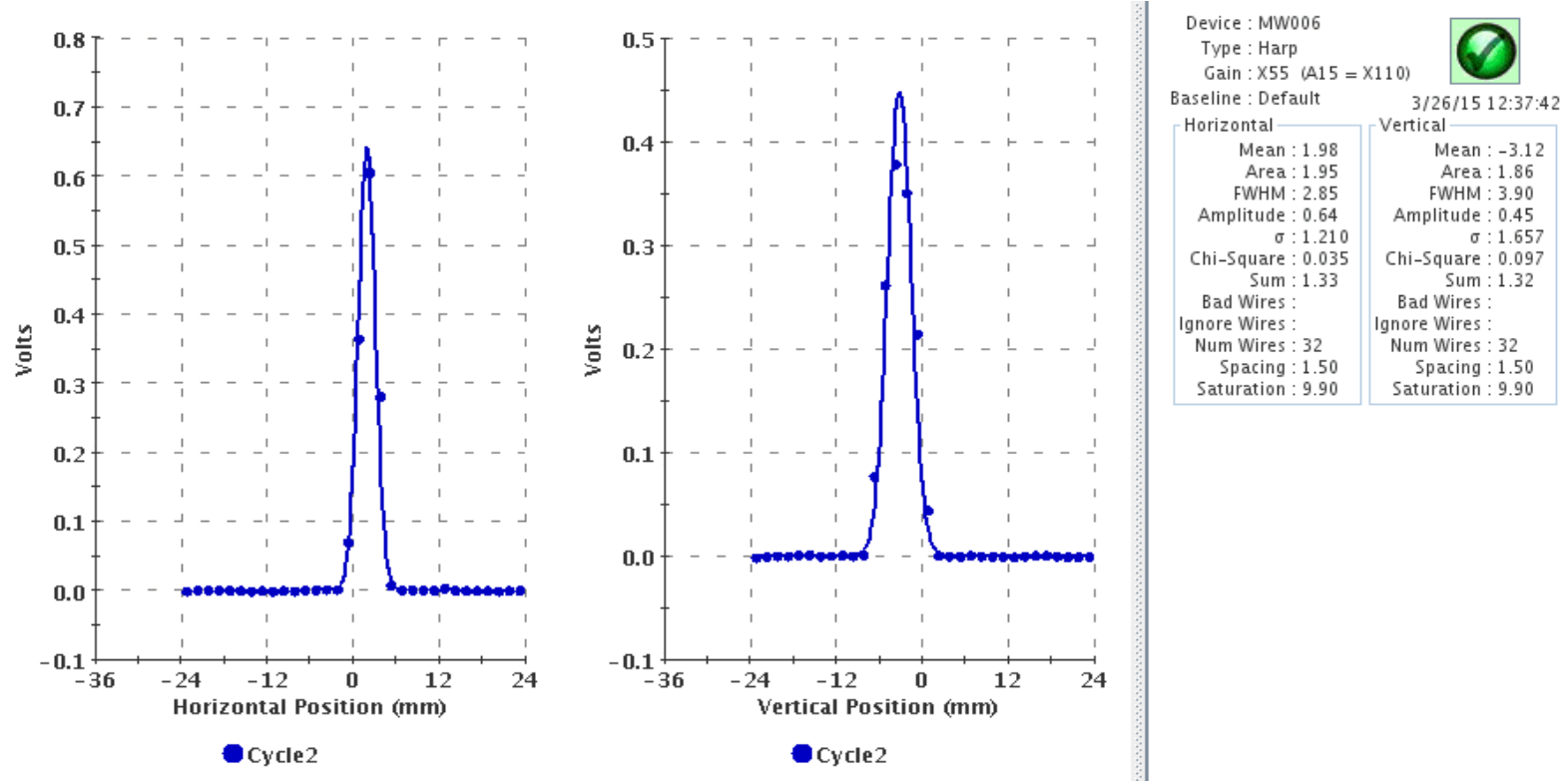

Figure 13: Profiles for scraped beam on BtA MW006 for typical running (Booster input is 9.0e11 and AGS late is 2.5e11).

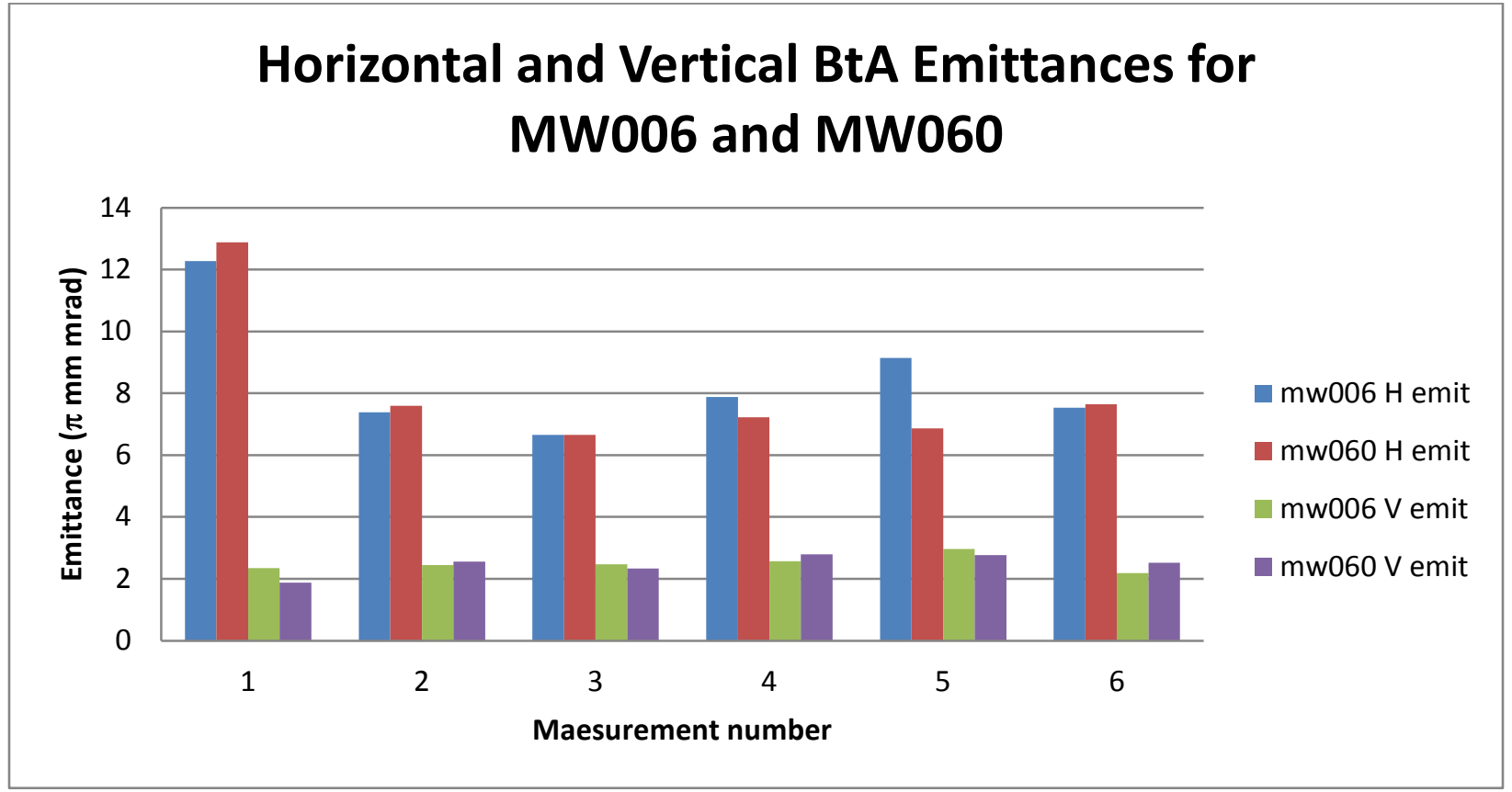

Figure 14: Comparison of Emittance measurements using MW006 and MW060 (Profiles were taken on Jan 11, May 19, 20, 21, 26, and Jun 17) 
Different proportions of vertical and horizontal scraping were used to see what effect they have on the AGS IPM flattop emittance. A study on Feb 17 showed that increasing the relative amount of horizontal to vertical scraping for a fixed AGS late intensity decreased the vertical flattop emittance and did not have a noticeable effect on the horizontal flattop emittance. The optimal proportion of horizontal to vertical scraping was about $2: 3 .{ }^{17}$

Figure 15 shows the ratio of the horizontal to vertical emittances on the 2 multiwires throughout the run. There is an extended period, mostly in April, where there aren't many measurements because of the multiwire complication.

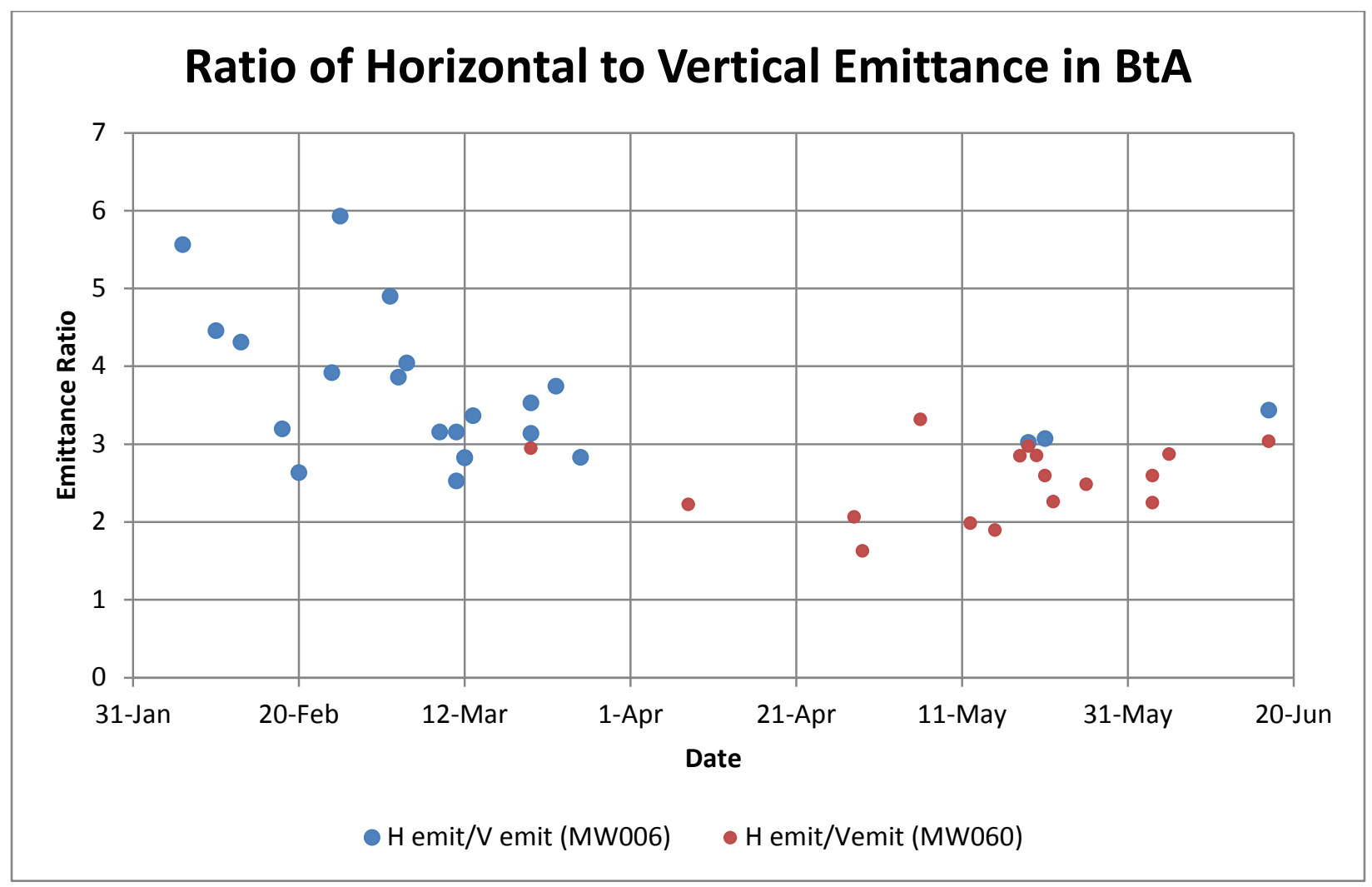

Figure 15: The ratio of the horizontal and vertical emittances is BtA as measured using multiwires mw006 (blue) and mw060 (red) through the 2015 run.

Figure 16 shows BtA emittance measurements from both MW006 and MW060 during the run with scraped beam (using the Gaussian fit option). The bottom plot shows the Booster input and AGS late when the data was taken. Note that from Apr 28 to May 18 Booster input was lower than normal (7 vs 9e11). Although it's not clear from the plot the intensity requirements for the AGS were lower during this time (PP-Au setup in RHIC) and so the source temperature was lowered. Perhaps related, the vertical BtA emittance does seem a bit higher during this time.

${ }^{17}$ Feb 17 Booster-AGS PP elog, 1722 entry 


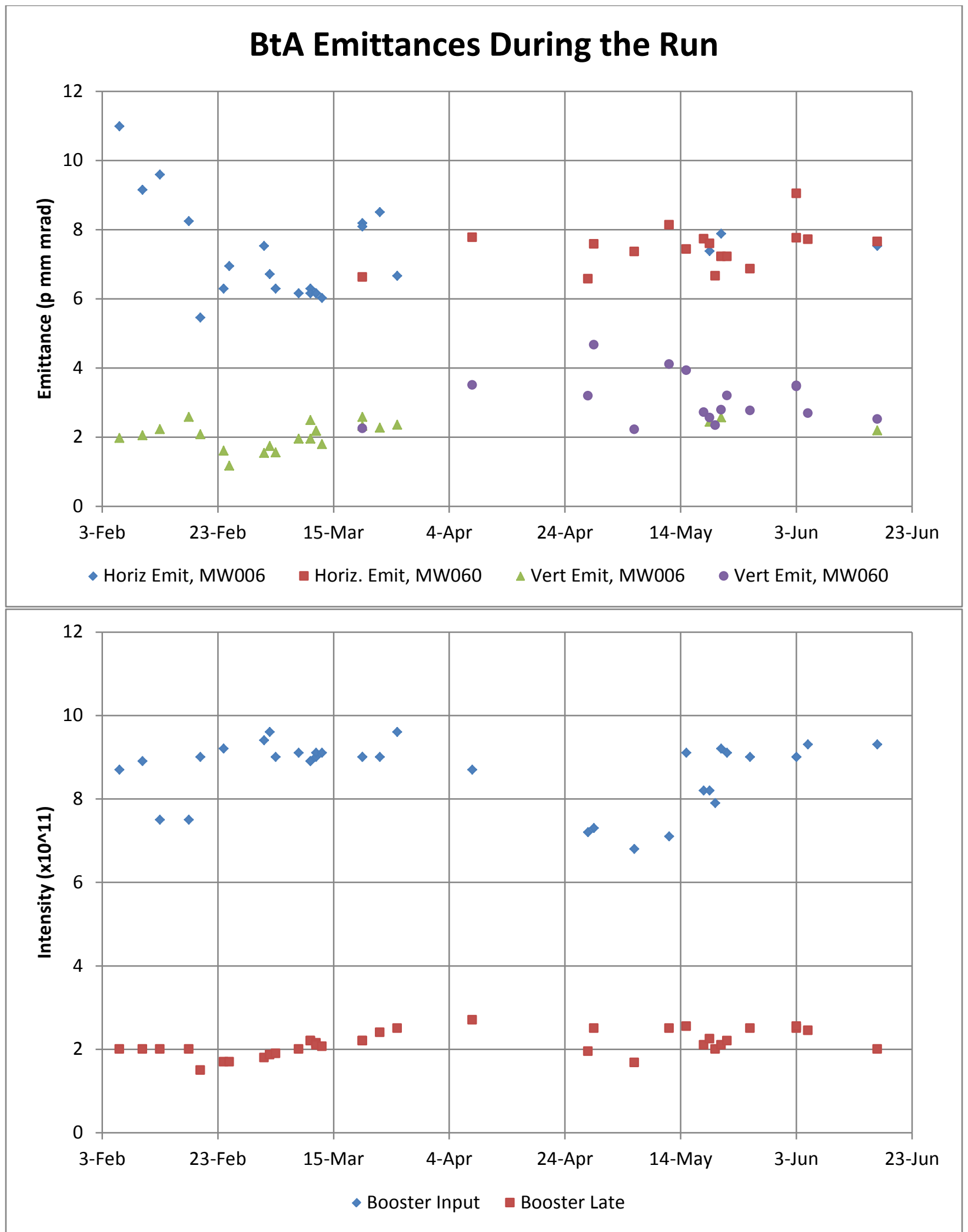

Figure 16: BtA emittances during the run using MW006 and MW060 (above) and Booster input and late intensities at the time of data taking (below). 


\section{The Tunes Early in the AGS cycle and the IPM flattop emittance}

I'll consider the part of the cycle before $0+\mathrm{Q}_{\mathrm{v}}=\mathrm{G} \gamma$ (hence forth to be known as $0+$, and similarly with the other intrinsic spin resonances) to be the early part of the cycle. Injection occurs around At $0+143 \mathrm{~ms}$ and $0+$ is at about At $0+262 \mathrm{~ms}$. At some point during this part of the cycle $\mathrm{Q}_{\mathrm{v}}$ is raised so that it will be in the spin tune gap by the time 0+ occurs. But this doesn't usually start to happen before At $0+200 \mathrm{~ms}$ or so. Experience has shown that the tunes during this part of the cycle are critical for emittance preservation.

There has been some question as to whether the tune paths before $0+$ are critical for avoiding depolarizing resonances such as the imperfection resonances at $\mathrm{G} \gamma=5,6$, etc. In order to avoid these resonances $Q_{v}$ would have to be close to 9 for it to fit within the spin tune gap. This can result in some beam loss at these low energies, and more importantly the values of $\mathrm{Q}_{\mathrm{v}}$ that seem to be required for emittance preservation during this part of the cycle are much lower than those required to fit within the spin tune gap. Additionally, emittance blowup seems to be kept to a minimum when the tunes are close to each other. And yet, theoretically, from a polarization perspective, keeping the tunes far from each other is preferable.

In years past, Qv has been raised several times so that it is in the spin tune gap for the above resonances. There has been no discernable effect on polarization. Nor has having the tunes close to, or passing through each other, been shown to effect the polarization. Consequently, when tuning this part of the cycle, there are no polarization related constraints on where the tunes can be placed except that the polarization should be checked when a substantial change is made. Smaller changes are made on a regular basis without necessarily checking the polarization.

Primarily during the first couple months of the run a lot of attention was given to adjusting the tunes during this part of the cycle to reduce the emittance mainly as viewed on the flattop with the IPM. The main issue is with vertical emittance growth. Years ago, with high intensity protons, it was clear that keeping Qv lower and keeping the tunes close together reduced the likelihood of coherent instabilities. It wouldn't be surprising if the same kind of mechanism were responsible for emittance growth here and that the solution in this case is the same.

The transfer from the Flat to Pulsed voltage banks (F to P transfer) of the main magnet also occurs during this time $(190 \mathrm{~ms})$ and is an additional complication. There are also jump quads firing around the time of the $\mathrm{F}$ to $\mathrm{P}$ transfer. But all this is nothing new.

Figures 17 and 18 show a series of tune scans ranging from nearly injection (145 ms) to $224 \mathrm{~ms}$ from Feb 13 that show the tune paths in the case where emittance growth has more or 
less been minimized. ${ }^{18}$ This is with the tune jumps on. They pulse between 187 and 191 ms and between 204 and $222 \mathrm{~ms}$. After these changes 3 polarization measurements were made and no drop in polarization was seen. ${ }^{19}$ It is not as though these tune paths did not change during the run as another tune scan from Mar 30 indicates that the tunes were close to each other but no longer crossing around 190 ms. However, the tunes were close to each other and $Q_{v}$ was not in the spin tune gap during this time in the cycle for this run. ${ }^{20}$

Several tune changes early in the cycle were made on previous days as well. Other changes were made early in the cycle too, such as adjustments to the radius. Table II shows the flattop emittance as measured by the IPM on those days. ${ }^{21}$ This data gives a sense of the effect of these tune changes on emittance. ${ }^{22}$

\begin{tabular}{|c|c|c|c|c|c|}
\hline Date & $\begin{array}{c}\text { AGS late } \\
\left(\mathrm{x} 10^{11}\right)\end{array}$ & $\begin{array}{c}\varepsilon_{\mathrm{h}} \\
(\pi \mathrm{mm} \text { mrad })\end{array}$ & $\begin{array}{c}\varepsilon_{\mathrm{V}} \\
(\pi \mathrm{mm} \text { mrad })\end{array}$ & $\sqrt{\varepsilon_{h}^{2}+\varepsilon_{v}^{2}} / \sqrt{2}$ & Polarization \\
\hline Feb 10 & 2.0 & 12 & 17.5 & 15.0 & \\
\hline Feb 10 & 2.0 & 10 & 16.5 & 13.6 & $72 \%$ \\
\hline Feb 11 & 2.0 & 11 & 16 & 13.7 & $73.5 \%$ \\
\hline Feb 12 & 1.5 & 11 & 16 & 13.7 & $73.0 \%$ \\
\hline Feb 13 & 2.0 & 11 & 15 & 13.2 & \\
\hline
\end{tabular}

Table II: Flattop IPM (RF off) emittances, intensity, and polarization measurements for the period between Feb $10^{\text {th }}$ and $13^{\text {th }}$.

\section{Polarization Variation during the Run}

Not unlike previous runs, this run was marked by periods of higher polarization as well as periods of less than stellar polarization. In this run though the periods of lower polarization perhaps seemed more protracted than in previous runs. As noted at the RHIC retreat, the periods of relatively low polarization were Mar 27-29, Apr 13-27, May 11-20, and Jun 5-21. It was also noted there that the low polarization in May was due to low source polarization and that the reasons for the low polarization during the other periods were unknown. ${ }^{23}$

There were many polarization measurements taken during the run. Many were taken as part of intensity scans and the like. Other measurements were taken during the course of operations. The

\footnotetext{
${ }^{18}$ Feb 13 Booster AGS PP elog 1656 entry

${ }_{19}$ The measurements made at the RHIC filling intensity (1.4e11 AGS late) were (75.7\%, 78.5\%, and 77\%). An additional measurement at 2.0e11 AGS late yielded 73.0\%. Feb 13 Booster AGS PP elog

${ }^{20}$ Mar 30 Booster AGS PP elog 1611 entry

21 Booster AGS PP elog

${ }^{22}$ More tune changes early in the cycle were made, most notably on Feb 25 and Mar 13. On Feb 25 the IPM measured $\varepsilon_{\mathrm{h}}=11$ and $\varepsilon_{\mathrm{v}}=16 \pi \mathrm{mm}$ mrad for $1.7 \mathrm{e} 11$, and on Mar 13 it measured $\left(\varepsilon_{\mathrm{h}}, \varepsilon_{\mathrm{v}}\right)=(10,15.7) \pi \mathrm{mm} \mathrm{mrad}$ for 2.2e11. .After the changes on Feb 25 the polarization was 68.9\%, and on Mar 13 with 2.2e11 3 measurements before the change were $72 \%, 73.3 \%$, and $73.1 \%$, and another three after the change were $69.8 \%, 67.6 \%$, and $73.2 \%$.

Booster AGS PP elog

${ }^{23}$ H. Huang, "Polarization in the Injectors", page 2.
} 
latter are the measurements I will concern myself with here. When the polarization was on the low side more of these polarization measurements were taken, so these measurements as a whole are biased towards lower polarization. It also turns out, that the problems with lower polarization
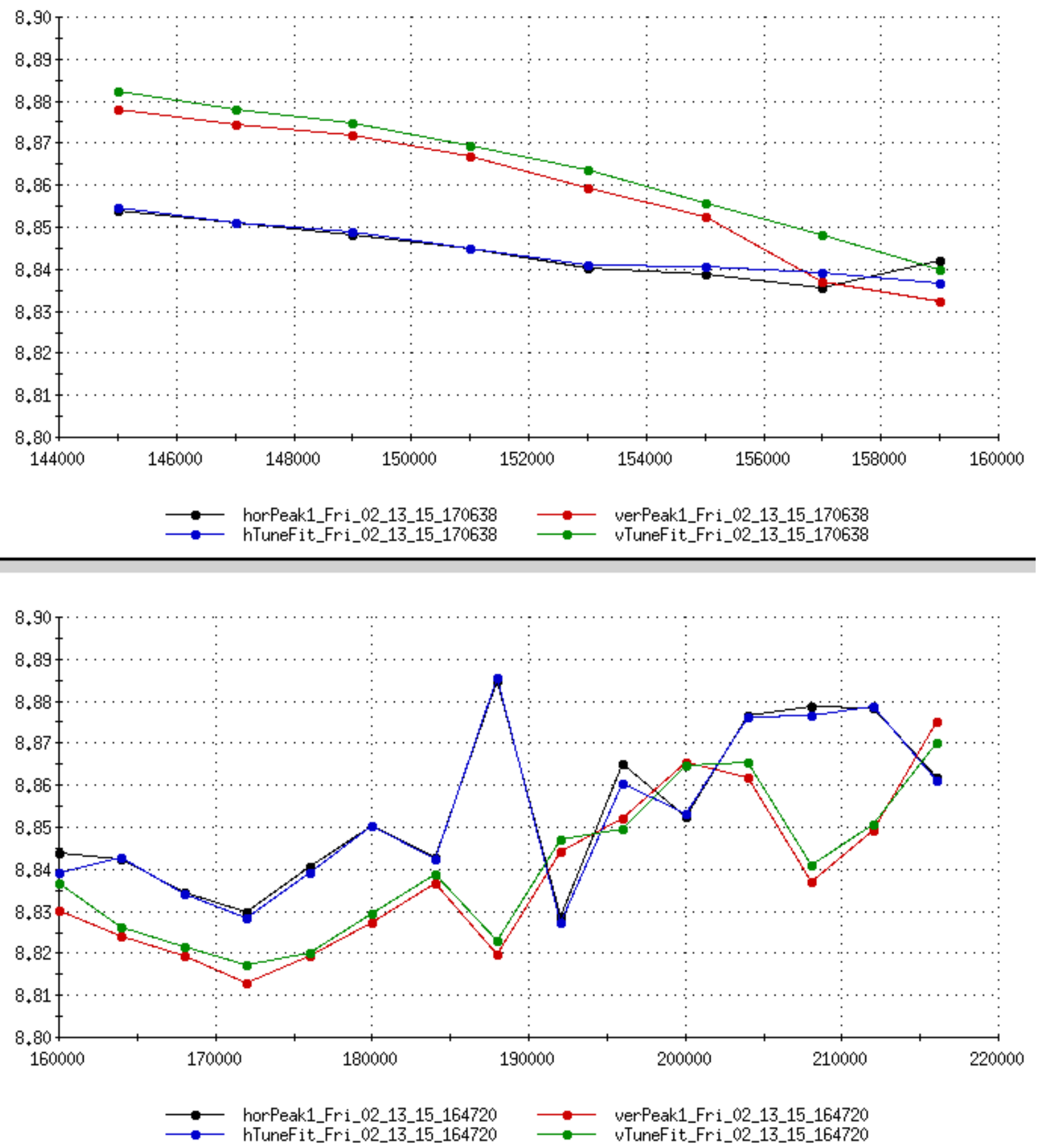

Figure 17: Tune scans from At0+145 ms to 216 ms illustrating the tune paths during the early part of the AGS cycle. Y-axis is tune, $x$-axis is time from At0 in ms. Scans were taken on Feb 13. 


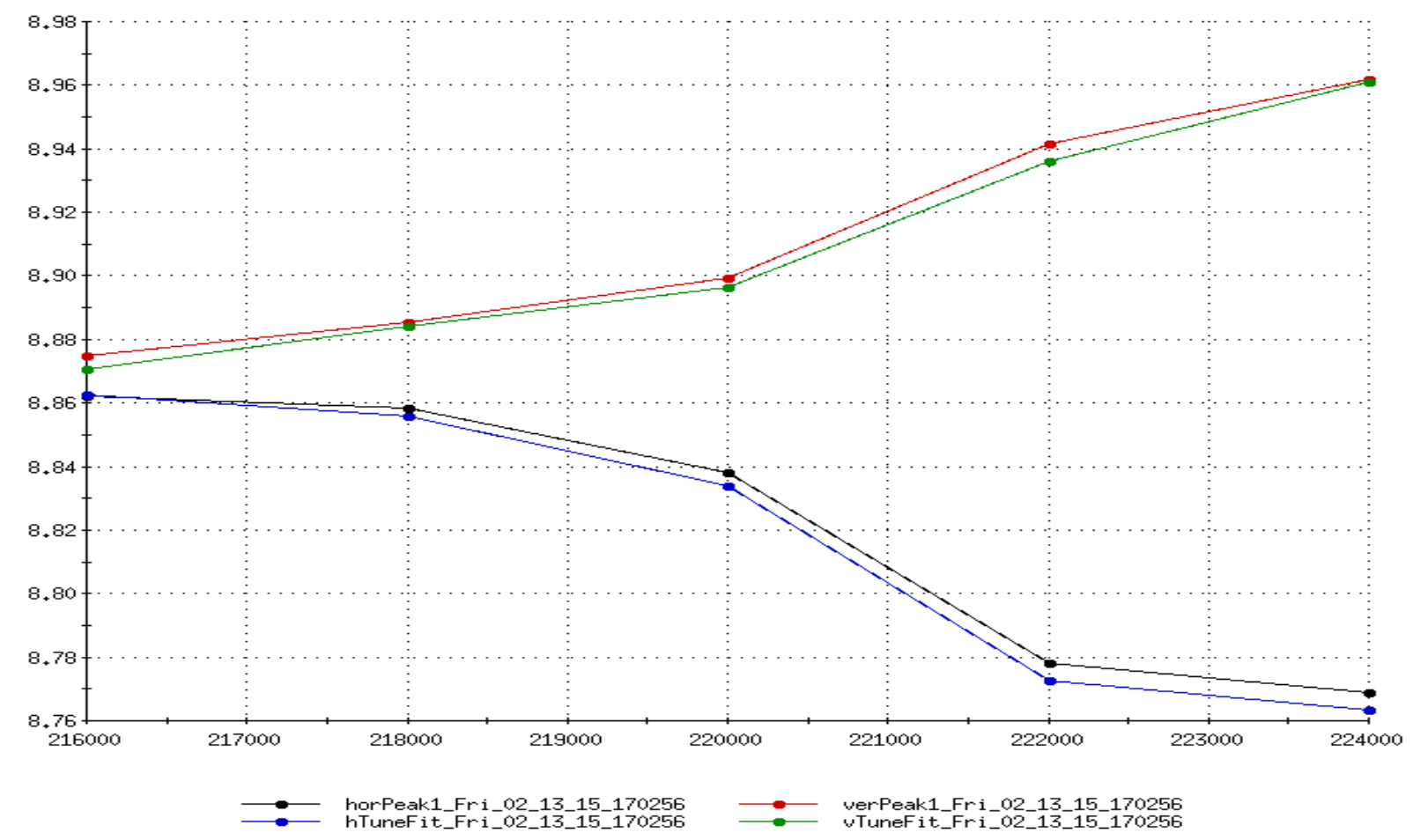

Figure 18: Tune scans from At0+216 ms to $224 \mathrm{~ms}$ illustrating the tune paths during the early part of the AGS cycle. Y-axis is tune, $x$-axis is time from At0 in ms. Scans were taken on Feb 13.

developed once we had reached higher intensities, so that this set of measurements is also biased towards higher intensity. This makes the intensity dependence look stronger than it would be otherwise. Figure 19 shows a subset of that polarization data associated with RHIC fills, which does not suffer from some of that bias. ${ }^{24}$ It also shows the same subset of data, but from Run 13 for comparison. Data point error bars have been left out because the plots are already too cluttered. In the fits to the data all of the points are taken to have the same weight and measurement uncertainty. ${ }^{25}$

Most of the measurements from Run 13 are clustered around an intensity of 2.0e11, whereas the data for Run 15 are more evenly distributed. There is also more data at higher intensities for Run 15. The data suggest that the average polarization in Run 15 was no lower than in Run 13. In fact, it looks like it was in general somewhat higher. The intensity dependence in Run 15 appears somewhat greater as indicated by the steepness of the linear fit to the data.

\footnotetext{
${ }^{24}$ This data was from obtained from the FDAView application. For Run 15 the agsPolT2 is used, and for Run 13 the agsPolT1 is used. A handful of measurements were removed from the Run 13 data because the polarization was very low and clearly something was wrong when the measurement was taken. It's also probably true that the polarization measurements associated with RHIC fills might be biased towards higher polarization, but this is probably the case in both Run 15 and Run 13.

${ }^{25}$ The average error in the Run 13 data is plus or minus $2.67 \%$, the standard deviation of these errors $\left(\sigma_{\text {err }}\right)$ is 1.21 $\%$. For Run 15, the average error is plus or minus $1.81 \%$ and $\sigma_{\text {err }}$ is $0.71 \%$. In Run 13 the error never goes below ${ }^{+} / .2 \%$, whereas the Run 15 data does not seem to have a hard minimum. Linear fits to the errors vs. intensity show that there is some intensity dependence (about -0.5\%/1e11). Higher intensities tend to have smaller errors. The intensity dependence is about 2.5 times greater in Run 13 than Run 15.
} 
However, the data suggests that the Run 15 polarization was on average at least no lower at the AGS late intensities used for RHIC than would've been expected for those intensities in Run 13.

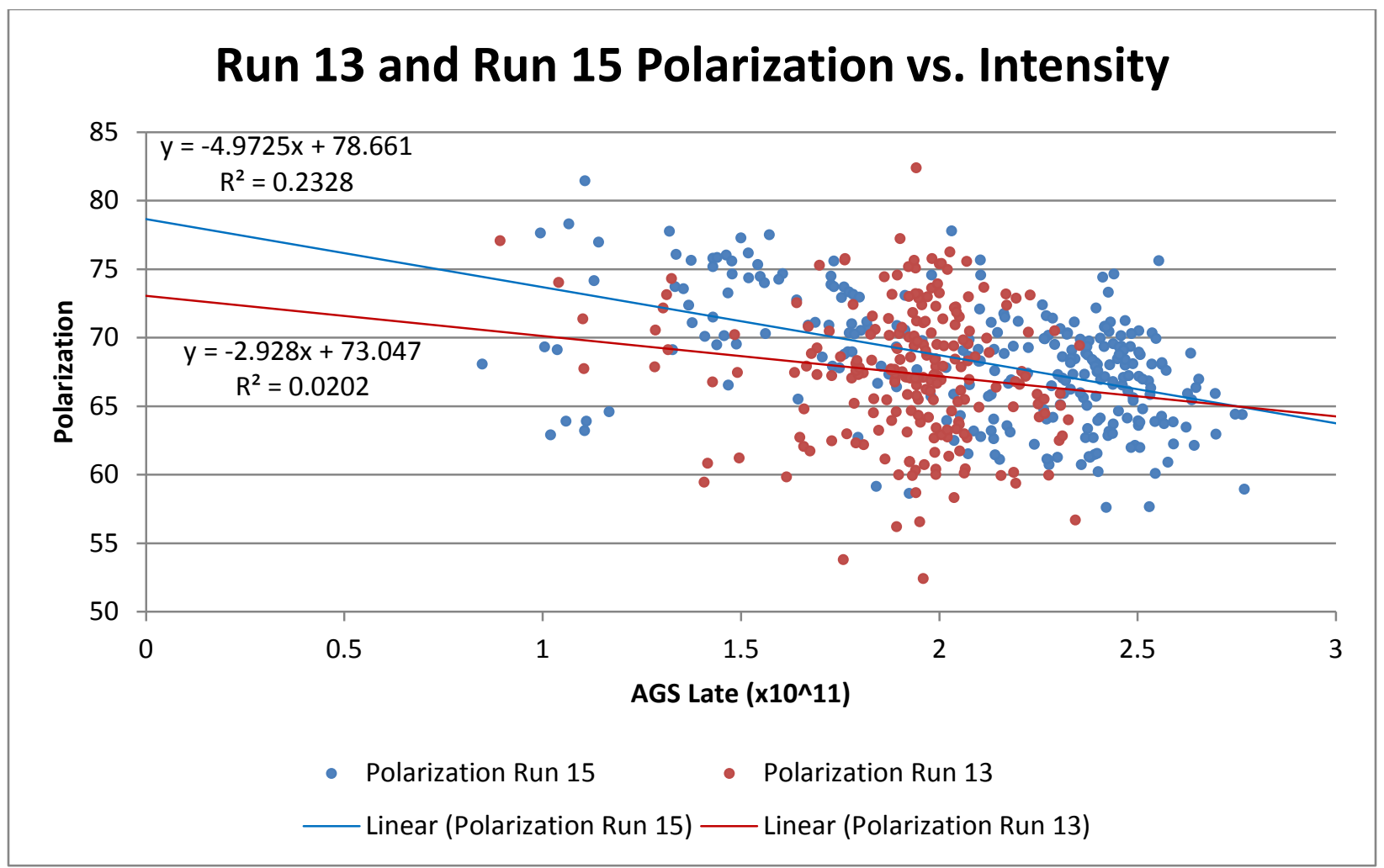

Figure 19: AGS Polarization vs. AGS late intensity associated with RHIC fills in Runs 13 and 15. Linear fits for both sets of data are included. Run 13 is in red, and Run 15 is in blue.

Figure 20 shows the same data but now the Run 15 data has been divided into 2 sets. In blue is the data from when the polarization was in the high state and in green from when it was in the low state (the dates mentioned above except for May 11-20, which is not included in either set of data). The slopes of the Run 15 high state and Run13 are quite similar. The Run 15 low state has a steeper intensity dependence.

All the data for the low polarization state is for intensities higher than 1.98e11. Figure 21 only shows the high state data for Run 15 with AGS late above 2e11 together with the low state data. It also shows only the Run 13 data for intensities above 2e11. One thing that's notable about the high state data is that there is no obvious intensity dependence. Another thing is that the low state Run 15 data is not as intensity dependent as the Run 13 data, and for intensities above 2.3e11 or so the linear fit of the Run 15 bad state data has a higher polarization than it does for Run 13.

As mentioned earlier, the cause of the variation is not understood. But was there something different from previous years about the low state that was responsible for the lower polarization, or was there something different from previous years in the high state that was responsible for the higher polarization? Although Run 13 was far from perfect, it's compared to 
Run15 in order to try to answer this question. Though far from conclusive given the uncertainties in these measurements the data suggest that the high polarization state is more different from Run 13 than the low state is, and that the low state also may have had slightly higher polarization than there would have been in Run 13 if the intensities we used this year were used then.

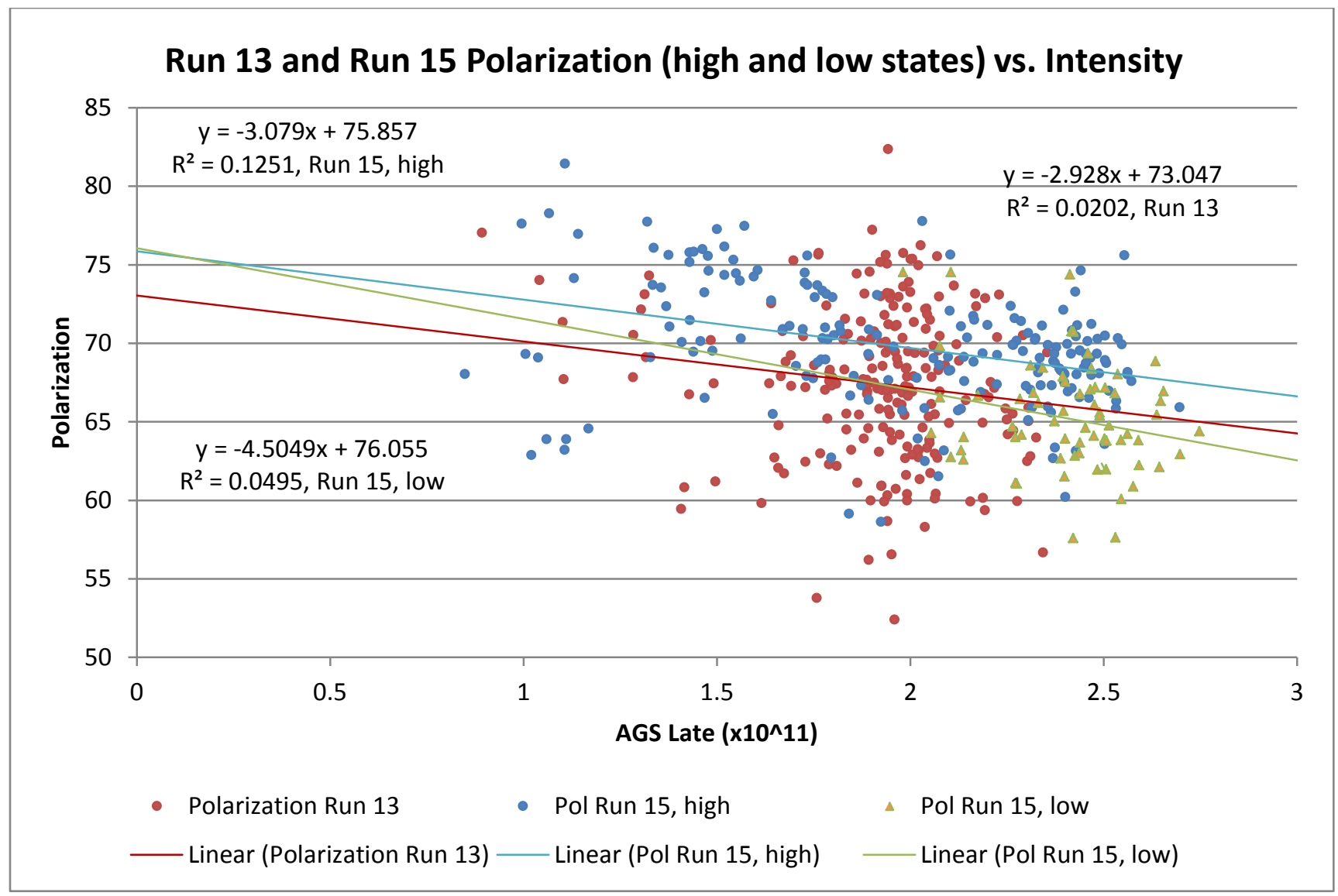

Figure 20: AGS Polarization vs. AGS late intensity associated with RHIC fills in Runs 13 and $\mathbf{1 5}$. The Run 15 data is divided into 2 states, "high" and "low". Linear fits for the 3 sets of data are included. Run 13 is in red, Run 15 (high state) is in blue, and Run 15 (low state) is in green.

\section{Dependence of Polarization on First Turn Bunch Width}

A study was conducted on May $8^{\text {th }}$ which looked at the dependence of polarization on the bunch width (a.k.a. length) and shape as seen on the first turn in the AGS. The polarization was compared at different intensities using a narrow, more peaked bunch and a wider, flatter bunch. Figure 22 gives a comparison of the 2 bunch types for the first turn in the AGS on the wall current monitor. ${ }^{26}$ The bunches were switched between the 2 states by detuning Linac (tank 4 phase) and Booster RF capture. The IPM flattop emittance was also measured. These transverse emittances were larger than usual, presumably because the source intensity was lower during this part of the run (around 7e11 vs. the more typical 9e11).

\footnotetext{
${ }^{26}$ Booster-AGS PP 2015 elog started May 7
} 


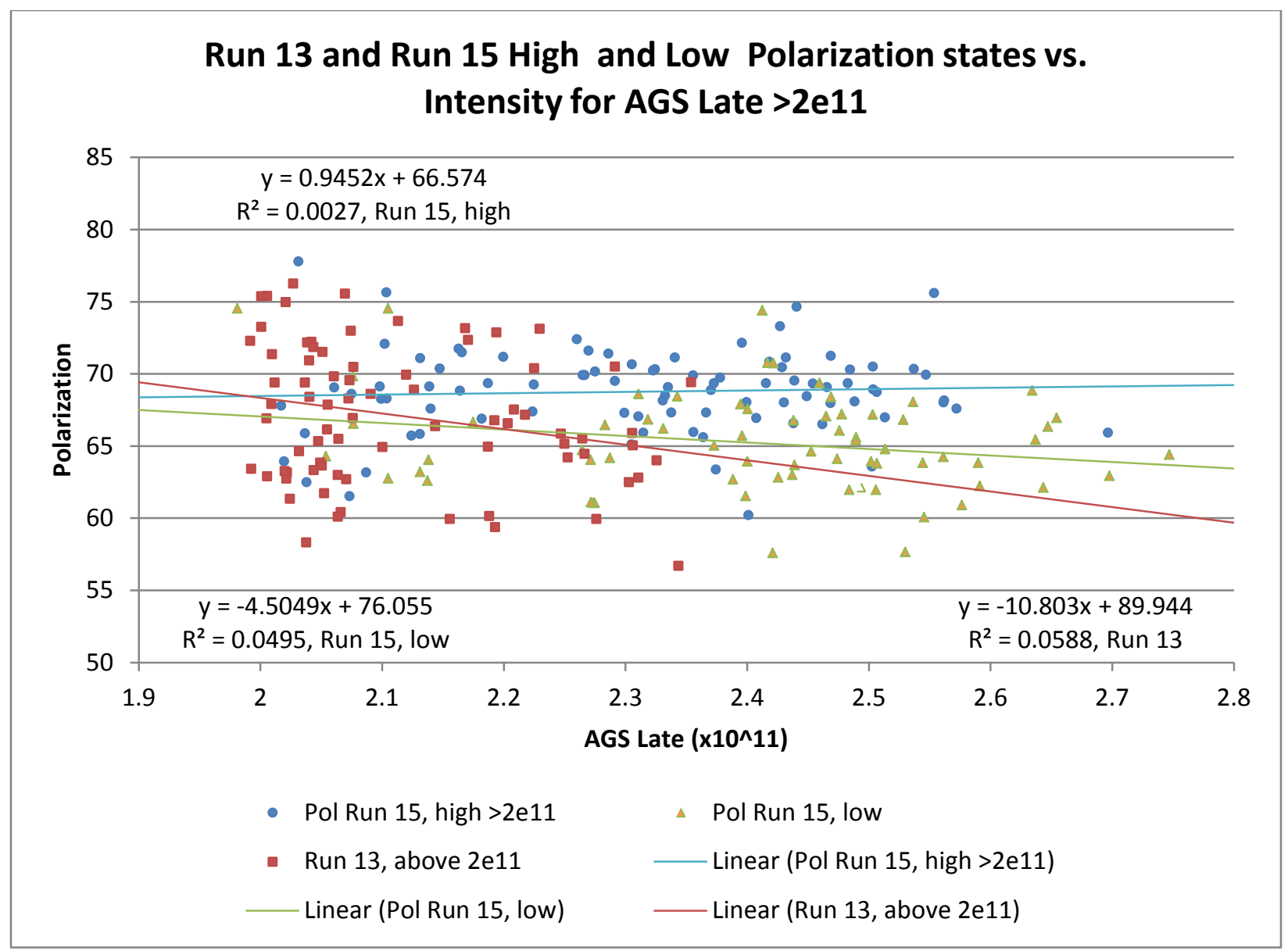

Figure 21: AGS Polarization vs. AGS late intensity associated with RHIC fills in Runs 13 and 15 . The Run 13 data is only for AGS late intensities >2e11. The Run 15 data is divided into high and low polarization states. For the high state, only the data for AGS late intensities >2e11 are used. Linear fits for the 3 sets of data are included. Run 13 is in red, and Run 15 high $>2 e 11$ is in blue, and Run 15 low is in green.

Figure 23 shows the results. Three polarization measurements were made for 5 different AGS late intensities ranging $1.5 \mathrm{e} 11$ to $3 \mathrm{e} 11$. The intensity was varied by changing the Booster scraping (both horizontal and vertical). The slopes of the linear fits are similar, but the polarization in the wider case is consistently several percent higher. This is somewhat surprising as in the past narrower bunches have been more prone to intensity dependent vertical blowup which one would think would be correlated with depolarization. If that were the case the narrower bunch case should show more intensity dependence and therefore a steeper slope.

Table III shows the IPM measurements on the flattop with the RF off. They show no sign of vertical blowup in the narrower bunch case, so at least that's consistent with the two cases having similar slopes. Perhaps it is because of the lower input intensity, and therefore larger initial transverse emittance that the vertical blowup did not occur. 


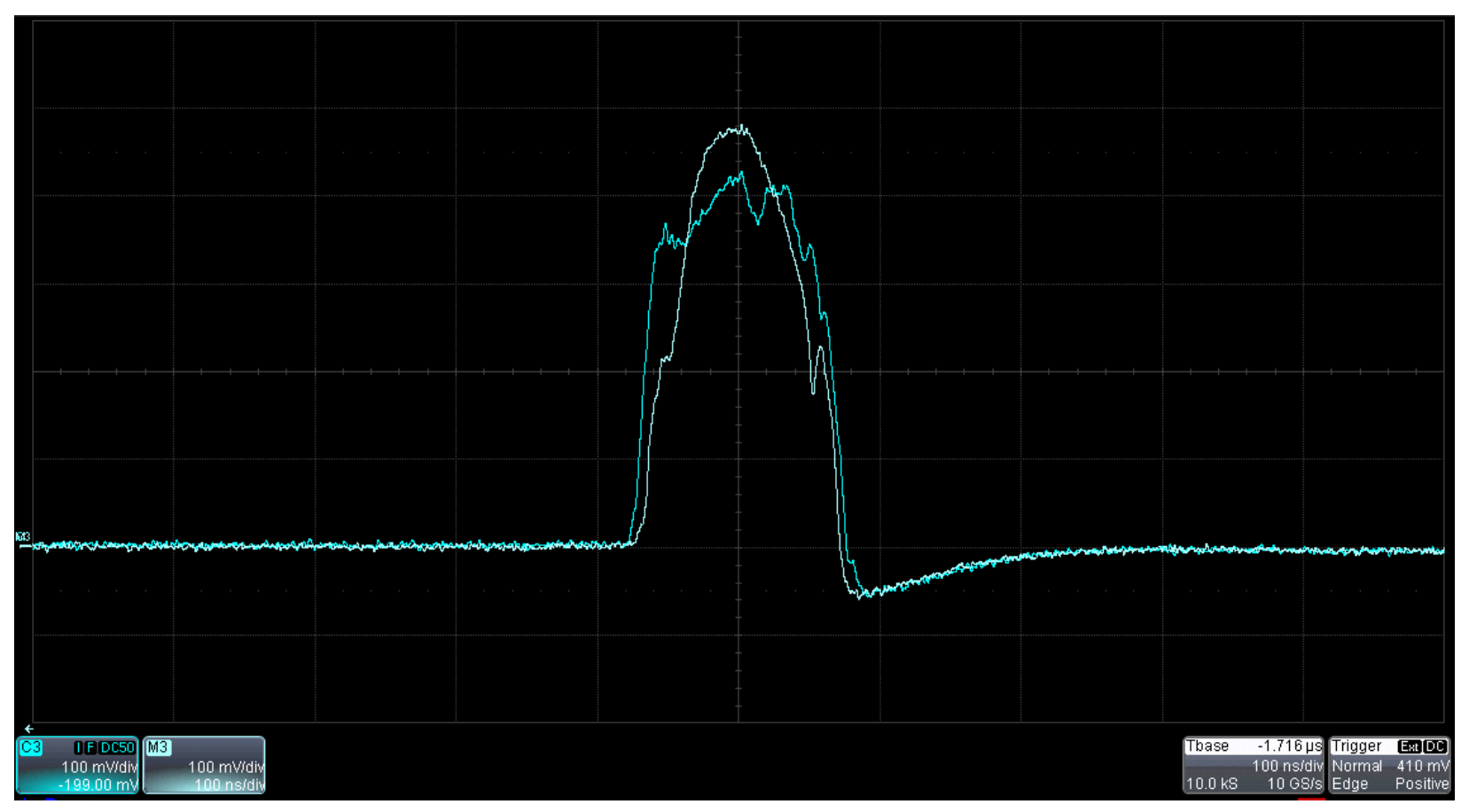

Figure 22: The first turn in the AGS in the 2 bunch states (wide and narrow). This is for an AGS late intensity of $2.4 \mathrm{e} 11$.

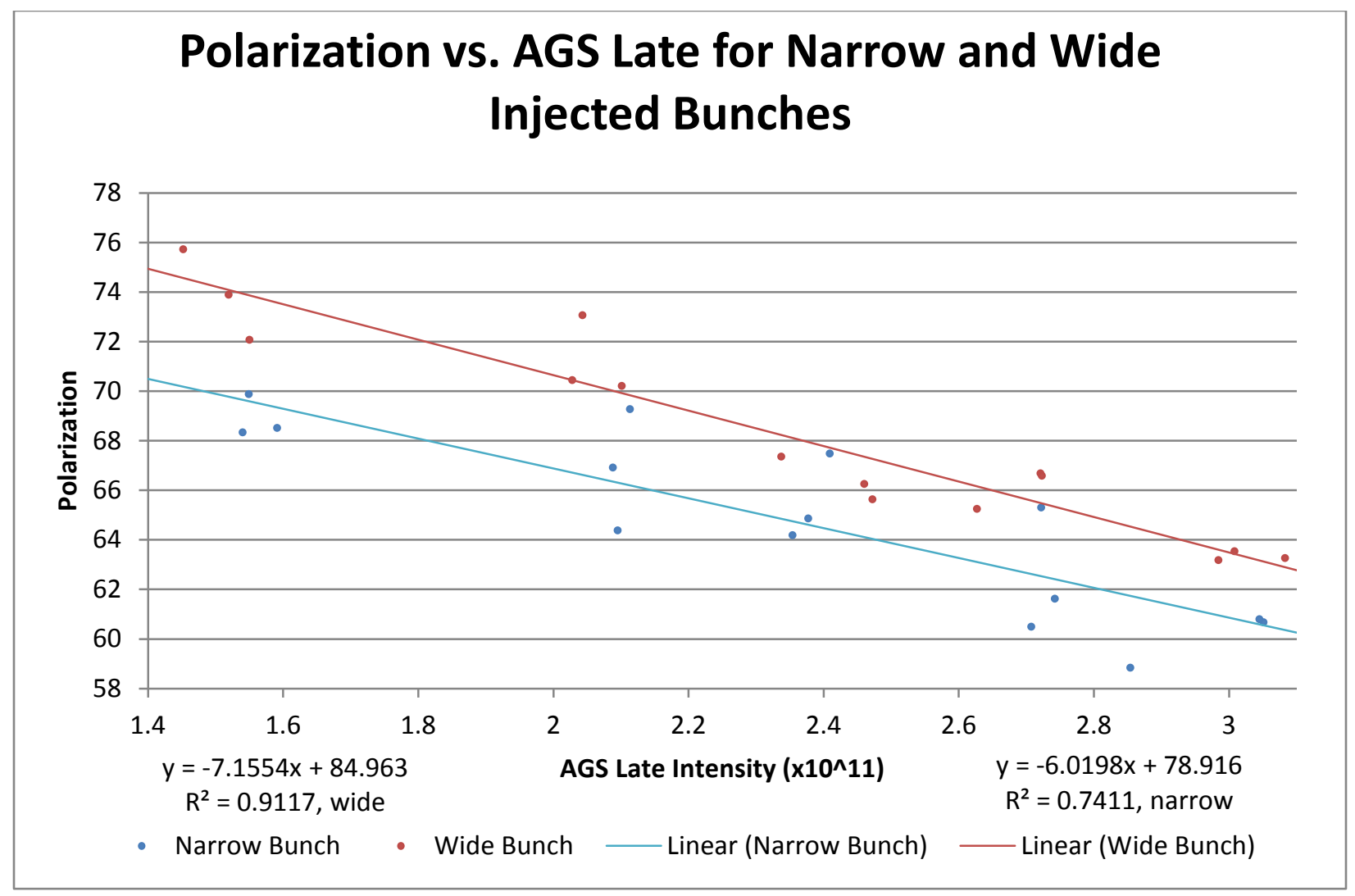

Figure 23: Polarization vs. AGS late for the 2 types of bunches. 


\begin{tabular}{|c|c|c|c|c|}
\hline AGS Late & Narrow $\boldsymbol{\varepsilon}_{\mathbf{h}}$ & Narrow $\boldsymbol{\varepsilon}_{\mathbf{v}}$ & Wide $\boldsymbol{\varepsilon}_{\mathbf{h}}$ & Wide $\boldsymbol{\varepsilon}_{\mathbf{v}}$ \\
\hline 1.5 & 11.5 & 13.5 & 12 & 15 \\
\hline 2.1 & 13 & 15 & 13 & 16 \\
\hline 2.4 & 14 & 17 & 14 & 17 \\
\hline 2.7 & 15 & 19 & 15 & 18.5 \\
\hline 3.0 & 15 & 20 & 16 & 20 \\
\hline
\end{tabular}

Table III: AGS Flattop IPM measurements with RF off for 'wide' and 'narrow' bunches at different AGS late intensities. AGS late is in units of $\times 10^{11}$ and emittance is in $\pi \mathrm{mm}$ mrad.

Unfortunately, bunch width measurements at extraction were not taken for the different cases. It could be argued that the polarimeter is somehow dependent on the bunch length, if not though this would seem to be a real effect. If it is real, then there may be depolarization effects associated with bunch width other than those associated with transverse emittance blowup which would therefore be hard to identify.

In this study the bunches were made wider by increasing the longitudinal emittance, at least at AGS injection. There are various scenarios one could imagine that would explain a nonintensity dependent effect which would improve the polarization for a smaller longitudinal emittance bunch. For example, the spread in $\mathrm{G} \gamma$ would be less at the horizontal resonances, so the tune jumps would be more effective. But it is hard to imagine a reason why bunches that have a larger longitudinal emittance would have a non-intensity dependent polarization improvement.

Given the results of this study though for much of the remainder of the run the bunches on the first turn were generally made wider (less peaked) than they could have been, but perhaps not quite as wide (and flat) as they were made for this study. After the study was done the extracted bunch length was left in the 28-29 ns range, which is barely different than what the bunch lengths had been up to that point in the run. Figure 24 shows the flattop bunch length measurements that were made during the run. Unfortunately, very few measurements were made in May and early June.

\section{AGS Flattop Emittances during the Run}

One rather substantial change in the setup, as it relates to IPM flattop emittance, occurred on Apr $1 .{ }^{27}$ It was noticed that lowering Qv early in the flattop and then raising it back up before extraction time resulted in a smaller vertical emittance and a somewhat larger horizontal one. Figure 25 shows the difference between the two states. The black trace is before the change and the red trace is after it. The flattop begins at around $600 \mathrm{~ms}$ and the RF is shut off at $1000 \mathrm{~ms}$. These traces are for an AGS late intensity of 2.2e11. The horizontal and vertical IPM data are not from the very same cycles.

\footnotetext{
${ }^{27}$ Booster AGS PP Mar 31 elog, entries from Apr 11813 to 1849
} 


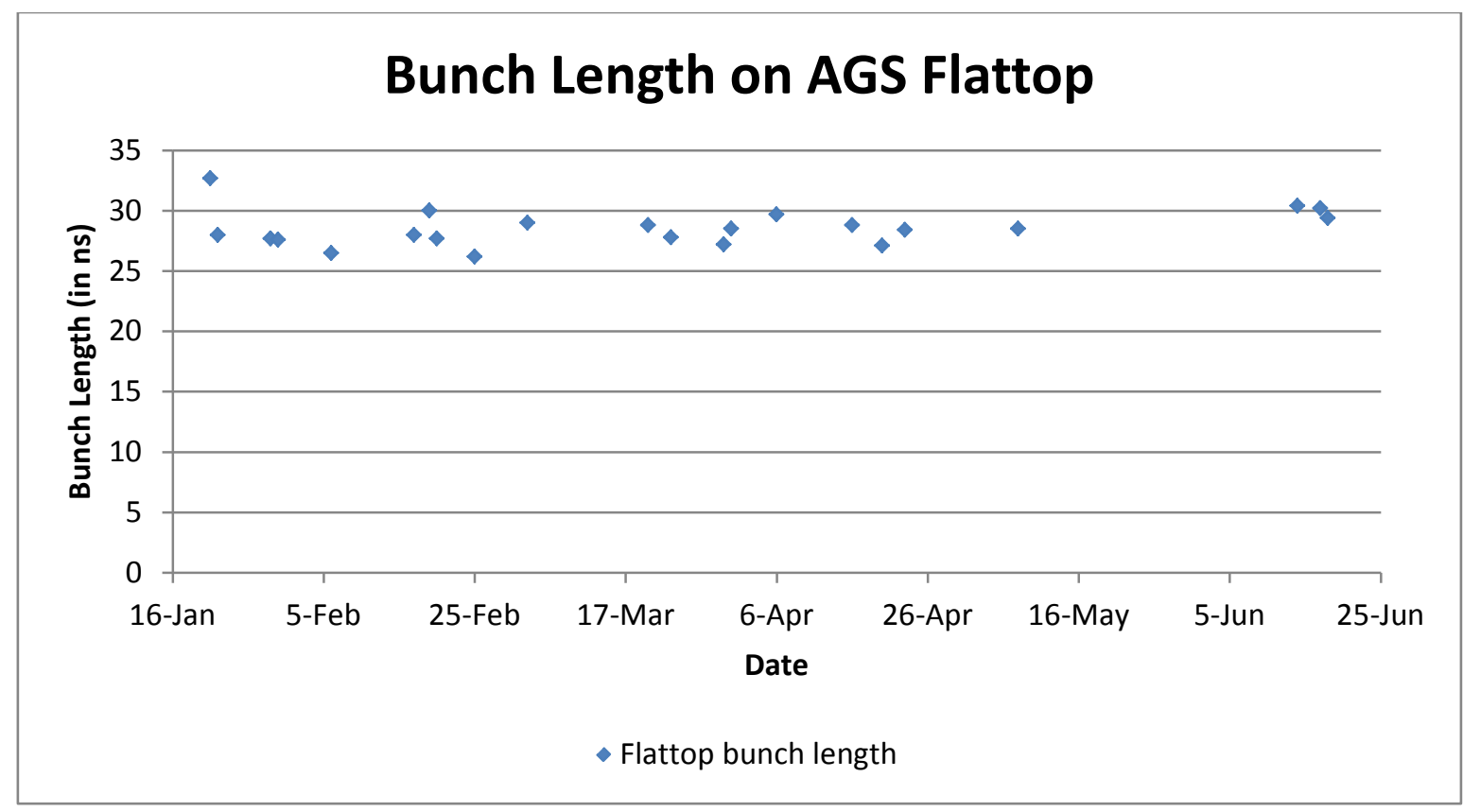

Figure 24: Full length of bunch on AGS flattop in nanoseconds vs day in the run.

These data indicate that $\varepsilon_{\mathrm{h}}$ is about $1 \pi \mathrm{mm}$ mrad larger (from 11 to $12 \pi \mathrm{mm}$ mrad) and $\varepsilon_{\mathrm{v}}$ is about $2.5 \pi \mathrm{mm}$ mrad smaller (from 17.0 to $14.5 \pi \mathrm{mm} \mathrm{mrad}$ ) after the change. This changes $\varepsilon_{\text {avg }}$ from 14.3 to $13.3 \pi$ mm mrad. ${ }^{28}$ This seemed like an obvious benefit, since careful inspection shows that the emittance change occurs before extraction time ( 930 ms). There was not a discernable effect on the AGS polarization though these changes were not left in. On Apr 2 finer adjustments to this setup change were made. The flattop emittance still responded similarly to the change, and there was no discernable effect on polarization, so that change was left in.

Figure 26 shows the $\varepsilon_{\mathrm{h}}, \varepsilon_{\mathrm{v}}, \varepsilon_{\mathrm{avg}}$, and AGS late during the run. Despite the changes on Apr 2 , when looking at the polarization through the run, it is hard to see if the change had an appreciable effect on $\varepsilon_{\text {avg. }}$. It does seem that the vertical was smaller afterwards, but it's not clear that the increase in $\varepsilon_{\mathrm{h}}$ didn't offset it completely. This is confusing, as there seemed to be a clear benefit from the change. Both plots have linear fits for $\varepsilon_{\mathrm{h}}, \varepsilon_{\mathrm{v}}$, and $\varepsilon_{\text {avg }}$ for before and after the Apr $2^{\text {nd }}$ change. It seemed like although $\varepsilon_{\mathrm{v}}$ remained reasonably constant through the remainder of the run (though there is a slight positive trend as well), that $\varepsilon_{\mathrm{h}}$ crept up, and that this is reflected in $\varepsilon_{\text {avg }}$ as well. AGS late over this period doesn't have an obvious trend, so it can't be blamed on that. A possible reason for it will be discussed below. Also of note is that although AGS late was increasing during the early part of the run, neither $\varepsilon_{\mathrm{h}}$ nor $\varepsilon_{\mathrm{v}}$ appear to be doing so. This is likely because improvements in the emittance were being made as the intensity was increased.

${ }^{28} \varepsilon_{\text {avg }}$ is the average emittance, $\sqrt{\varepsilon_{h}^{2}+\varepsilon_{v}^{2}} / \sqrt{2}$. 


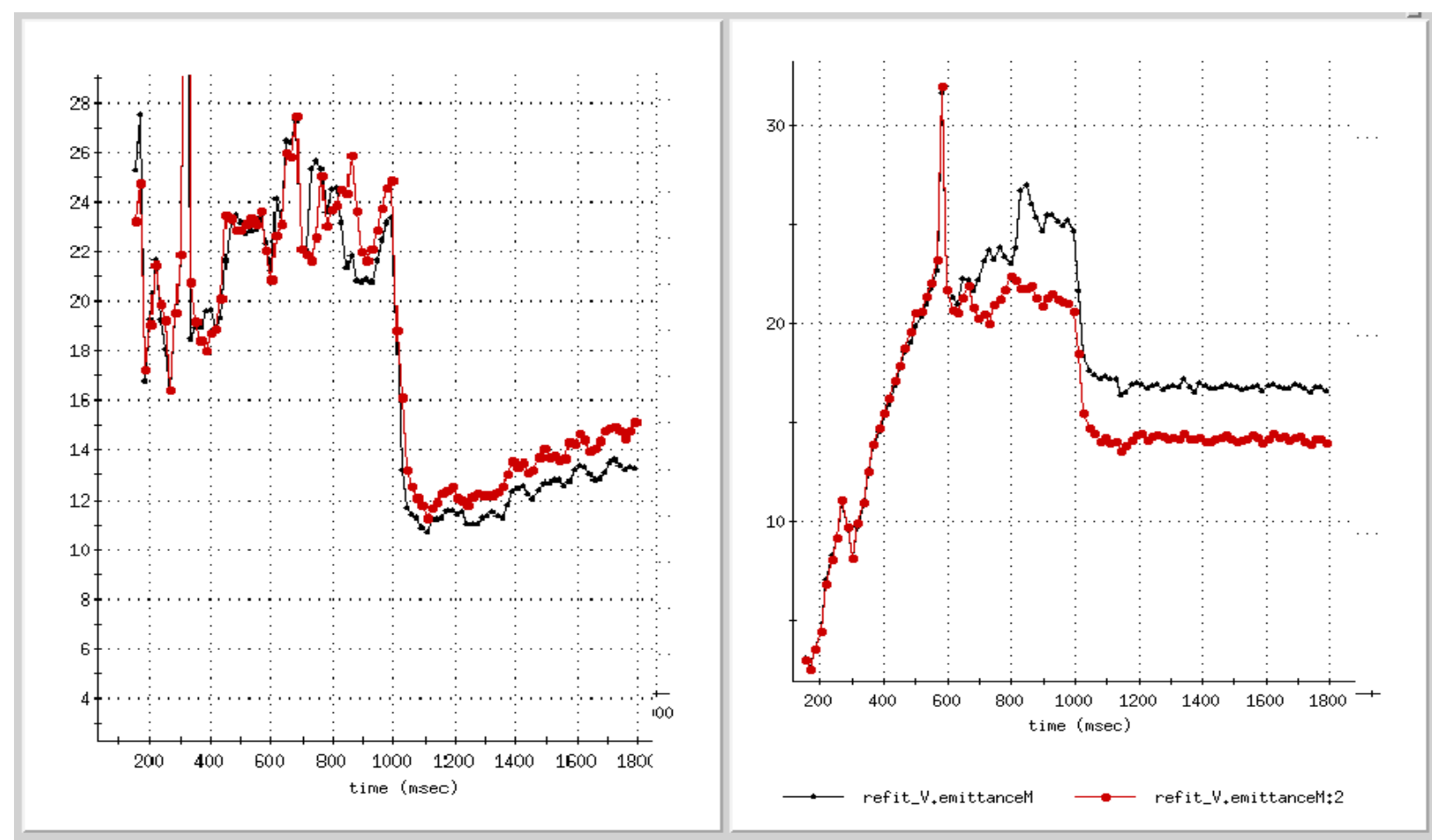

Figure 25: AGS IPM horizontal (left) and vertical (right) measurements with Qv shifted early on flattop (red) and not shifted (black). The AGS late intensity is 2.2e11.

The horizontal IPM measurement does not account for the dispersion component of the horizontal width though, so one possibility is that the increase in the measured $\varepsilon_{\mathrm{h}}$ during the latter part of the run was due to an increase in $\Delta \mathrm{p} / \mathrm{p}$, or longitudinal emittance $\left(\varepsilon_{\text {long }}\right)$. The dispersion at the horizontal IPM is about $2 \mathrm{~m} .{ }^{29}$ The IPM measurement is of the normalized emittance, $\varepsilon_{\mathrm{h}}=\gamma^{*} \mathrm{x}^{2} / \beta$ where $\mathrm{x}$ is the $95 \%$ half width of the fitted Gaussian. The relativistic factor $\beta_{\text {rel }}$ is essentially 1 at flattop energy (so it is left out) and $\gamma$ is about 25.4. Using $\beta=19 \mathrm{~m}$, an $\mathrm{x}$ of only $2.87 \mathrm{~mm}$ is required to produce an $\varepsilon_{\mathrm{h}}$ of $11 \pi \mathrm{mm}$ mrad. $\varepsilon_{\text {long }}$ was measured to be about 1.01 ev-sec for a bunch length of 28.4 ns. $^{30}$

With the nominal flattop voltage this corresponds to a $\Delta \mathrm{p} / \mathrm{p}$ of a $1.00 \mathrm{e}-3$ and a dispersion contribution to $\mathrm{x}$, called $\mathrm{x}_{\mathrm{d}}$, of $2.00 \mathrm{~mm}$. $\varepsilon_{\mathrm{h}}$ is measured after the RF voltage is shut off, but since the RF is shut off immediately, I'm assuming that $\Delta \mathrm{p} / \mathrm{p}$ is the same after it is off. Using $\left.\mathrm{x}=\left(\mathrm{x}_{\mathrm{o}}{ }^{2}+\mathrm{x}_{\mathrm{d}}\right)^{2}\right)^{0.5}$ gives an 'optical' width of $2.06 \mathrm{~mm}$, called $\mathrm{x}_{\mathrm{o}}$, for a measured $\varepsilon_{\mathrm{h}}$ of $11 \pi \mathrm{mm} \mathrm{mrad}$. So, the optical and dispersion components are about the same size. An increase in the dispersion component of $0.3-0.4 \mathrm{~mm}$ is all that's required to raise the measured $\varepsilon_{\mathrm{h}}$ from 11 to $13 \pi \mathrm{mm}$ $\mathrm{mrad}$. This corresponds to an increase in $\Delta \mathrm{p} / \mathrm{p}$ of only about $0.15 \mathrm{e}-3$, which corresponds to an increase in bunch length of about 4 ns (for an initial bunch length of $28.4 \mathrm{~ns}$ ).

\footnotetext{
${ }^{29}$ Proton Emittance Measurements in The Brookhaven AGS, H. Huang et al., pg. 494

30 Booster AGS PP elog Apr 231458 entry
} 

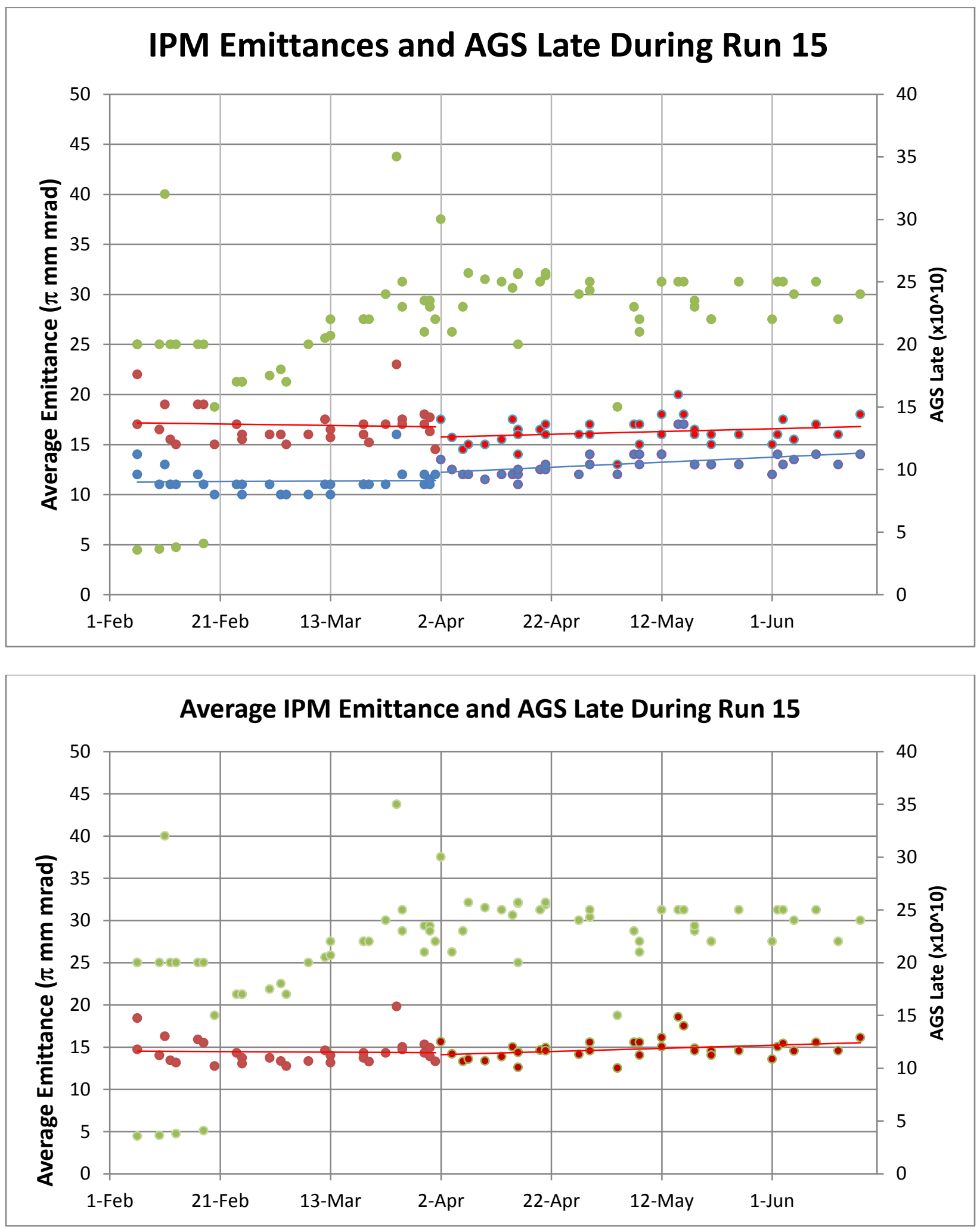

Figure 26: $\varepsilon_{\mathrm{h}}, \varepsilon_{\mathrm{V}}, \varepsilon_{\mathrm{avg}}$, and AGS late during Run 15. The top plot shows $\varepsilon_{\mathrm{h}}$ (red), $\varepsilon_{\mathrm{V}}$ (blue) and AGS Late (green), and the bottom shows $\mathcal{E}_{\mathrm{avg}}$ (red) and AGS Late (green). In both plots the left y-axis is for emittance and the right y-axis is for AGS Late $\left(\mathrm{x} 10^{10}\right)$. There are two linear fits each for $\varepsilon_{\mathrm{h}}, \varepsilon_{\mathrm{V}}$, and $\varepsilon_{\mathrm{avg}}$. The 2 sets of fits are for the data up to Apr 1 and for Apr 2 to the end of the run. 
Figure 24 shows that the bunch lengths measured during the run do vary by something like this amount. And since there was some attempt to make the bunches longer during the latter part of the run it seems quite possible that this may account for the increase in measured $\varepsilon_{\mathrm{h}}$ during that time. It also raises the question as to whether or not the polarization measurement is sensitive to the horizontal width on this level as that could account for the observation that wider injected bunches result in a higher measured polarization with no intensity dependence . Also, the actual $\varepsilon_{\mathrm{h}}$, corresponding to an optical width of $2.06 \mathrm{~mm}$ would be 5.7 instead of the measured $11 \pi \mathrm{mm}$ mrad. Additionally, if $\varepsilon_{\mathrm{h}}$ were actually 0 , the measured $\varepsilon_{\mathrm{h}}$ would still be about $5.4 \pi$ mm mrad for a $\Delta \mathrm{p} / \mathrm{p}$ of $1 \mathrm{e}-3$.

In light of this, what can be said about the observed change in $\varepsilon_{\mathrm{h}}$ and $\varepsilon_{\mathrm{v}}$ after the $\mathrm{Q}_{\mathrm{v}}$ shift? The measured $\varepsilon_{\mathrm{h}}$ increased by about $1 \pi \mathrm{mm}$ rad from 11 to $12 \pi \mathrm{mm} \operatorname{mrad}$. For $\Delta \mathrm{p} / \mathrm{p}=1 \mathrm{e}-3$ this corresponds to a change in $\mathrm{x}_{0}$ from 2.06 to $2.24 \mathrm{~mm}$. The actual $\varepsilon_{\mathrm{h}}$ for $\mathrm{x}_{0}=2.06 \mathrm{~mm}$ would be 5.7 $\pi \mathrm{mm}$ mrad and for $\mathrm{x}_{0}=2.24 \mathrm{~mm}$ it would be $6.7 \pi \mathrm{mm}$ mrad. This still corresponds to an increase of about $1 \pi \mathrm{mm}$ mrad. After the shift $\varepsilon_{\text {avg }}$ is still smaller by about $1 \pi \mathrm{mm}$ mrad (going from 12.7 to $11.3 \pi \mathrm{mm}$ mrad).

Given the IPM's wire spacing of $1.27 \mathrm{~mm}$ from wire center to center ${ }^{31}$ changes in $\varepsilon_{\mathrm{h}}$ (and $\varepsilon_{\mathrm{v}}$ ) on this level must be very hard to resolve. It would not be surprising if the result of the $\mathrm{Q}_{\mathrm{v}}$ shift was merely to mix the emittances of the 2 planes without actually reducing $\varepsilon_{\text {avg. }}$ Still though favoring an emittance reduction in the vertical seems to be the sensible thing to do. Yet, especially given the actual $\varepsilon_{\mathrm{h}}$, it appears that $\varepsilon_{\mathrm{v}}$ is 2 or 3 times larger than $\varepsilon_{\mathrm{h}}$ on the flattop. This has been the case in previous years as well. Given that $\varepsilon_{\mathrm{v}}$ is typically much smaller than $\varepsilon_{\mathrm{h}}$ in BtA, this would suggest that the emittances are somehow mixing or even flipping in the AGS. The period during the early part of the cycle where the tunes are highly coupled (see Figure 17) would seem to be the prime candidate for where this occurs, but regardless that configuration seems to lead to a lower $\varepsilon_{\mathrm{v}}$ and $\varepsilon_{\mathrm{avg}}$. However, as noted earlier, various horizontal to vertical scraping ratios have been explored and a solution that yields a smaller $\varepsilon_{\mathrm{v}}$ on flattop has not been found.

\section{Vertical Emittance Blowup Around 0+}

On Apr 3 vertical emittance blowup was observed on the IPM around 0+ (at 262-263 $\mathrm{ms}$ ). There was no beam loss associated with this blowup. Lowering $\mathrm{Q}_{\mathrm{v}}$ by about 0.002 to 8.9838 from 260 to $265 \mathrm{~ms}$ stopped this from occurring. The AGS late intensity was about 2.1e11. Polarization measurements were made, and it looked like it might be low, so $\mathrm{Q}_{\mathrm{v}}$ was eventually raised up to 8.9853. After this adjustment the setpoint was actually 0.0005 higher than where it

\footnotetext{
${ }^{31}$ Instrumentation wiki article on the AGS IPM
} 
was before the blowup started happening, but the polarization was good and the blowup was no longer happening. ${ }^{32}$ Looking back at the elog, typical $\mathrm{Q}_{\mathrm{v}}$ values around $0+$ appear to have been in the 8.985 to 8.986 range up to this point in the run. ${ }^{33}$

On Apr 4 it started happening again when $\mathrm{Q}_{\mathrm{v}}$ was raised from 8.9853 to as high as 8.9878 in an effort to improve polarization, but then it was lowered back to 8.9853 because of the vertical blowup. Despite lowering $\mathrm{Q}_{\mathrm{v}}$ back to 8.9853 the polarization looked good (74.5\% at 2e11 AGS Late). ${ }^{34}$

On Apr 6 the blowup was observed again. Qv jumps up at $261 \mathrm{~ms}$ when the tune jump quads fire, and jumps back down around $265 \mathrm{~ms}$. It was noted that raising $\mathrm{Q}_{\mathrm{v}}$ from 260 to $265 \mathrm{~ms}$ causes large oscillations on a vertical BPM as viewed on a scope. ${ }^{35}$ At first it was thought that the vertical kick the beam gets at $261 \mathrm{~ms}$ from the tune jump might be responsible for the blowup. However, it was soon noticed that even with significantly smaller oscillations the same amount of blowup can still occur. Figure 27 shows the vertical IPM with RF off with and without the blowup. The measurement at $252 \mathrm{~ms}$ is the same in both cases, but the one at $268 \mathrm{~ms}$ shows the blowup.

The top trace in Figure 28 shows the vertical BPM with blowup occurring $\left(Q_{v}\right.$ raised high), the middle trace shows it without blowup occurring (lower $\mathrm{Q}_{\mathrm{v}}$ ), and the bottom trace is another case where blowup is occurring that was noticed about 20 minutes later. The value of $\mathrm{Q}_{\mathrm{v}}$ in the latter case is not known. The amplitude of the noise on the traces is about 0.2 boxes (peak to peak). Given this the amplitude of the oscillation in the top trace is about 1.0 box, and about 0.6 boxes in the middle and bottom traces.

One puzzling thing is, since the same amount of blowup can occur in either the top or bottom cases, and the middle trace, where it doesn't occur has about the same amplitude oscillation as the bottom trace, it would seem that these vertical oscillations may not be the source of it. And if they are not the source of it, then what is? I would think that such emittance blowup would be associated with some kind of vertical coherent oscillation.

A BPM would not be able to resolve a coherent oscillation that leaves the center of mass of the bunch unchanged. ${ }^{36}$ The damper would not be able to damp that type of oscillation out either since it only acts on the center of mass of the bunch. One could imagine that such an oscillation might sometimes occur and cause emittance growth and other times damp out before

\footnotetext{
32 Booster AGS PP Apr 2 elog entries from Apr 3 1642-1728.

${ }_{33}$ On Apr 1 Qv was measured at 263 ms and was 8.9858. Mar 31 Booster AGS PP elog, Apr 10007 entry.

${ }^{34}$ Booster AGS PP elog Apr 4 1945-Apr 50336 entries.

35 Since Qv is so close to an integer, when viewed at a particular location in the ring betatron oscillations are slow enough that they can be seen on a BPM even though the BPMs were designed only to measure the closed orbit.

${ }^{36}$ For example, certain modes of the head-tail instability can cause the leading part of the bunch to move transversely in one direction, and the trailing edge to move by the same amount in the opposite direction. In that case the average position of the bunch doesn't change. For example the $m=1$ mode. See pg. 121 of "Handbook of Accelerator Physics and Engineering". A. Chao.
} 
emittance growth occurs. In the latter case, there could still be oscillations while passing through $0+$ that could cause depolarization but there would be no evidence of it on the diagnostics we typically use.

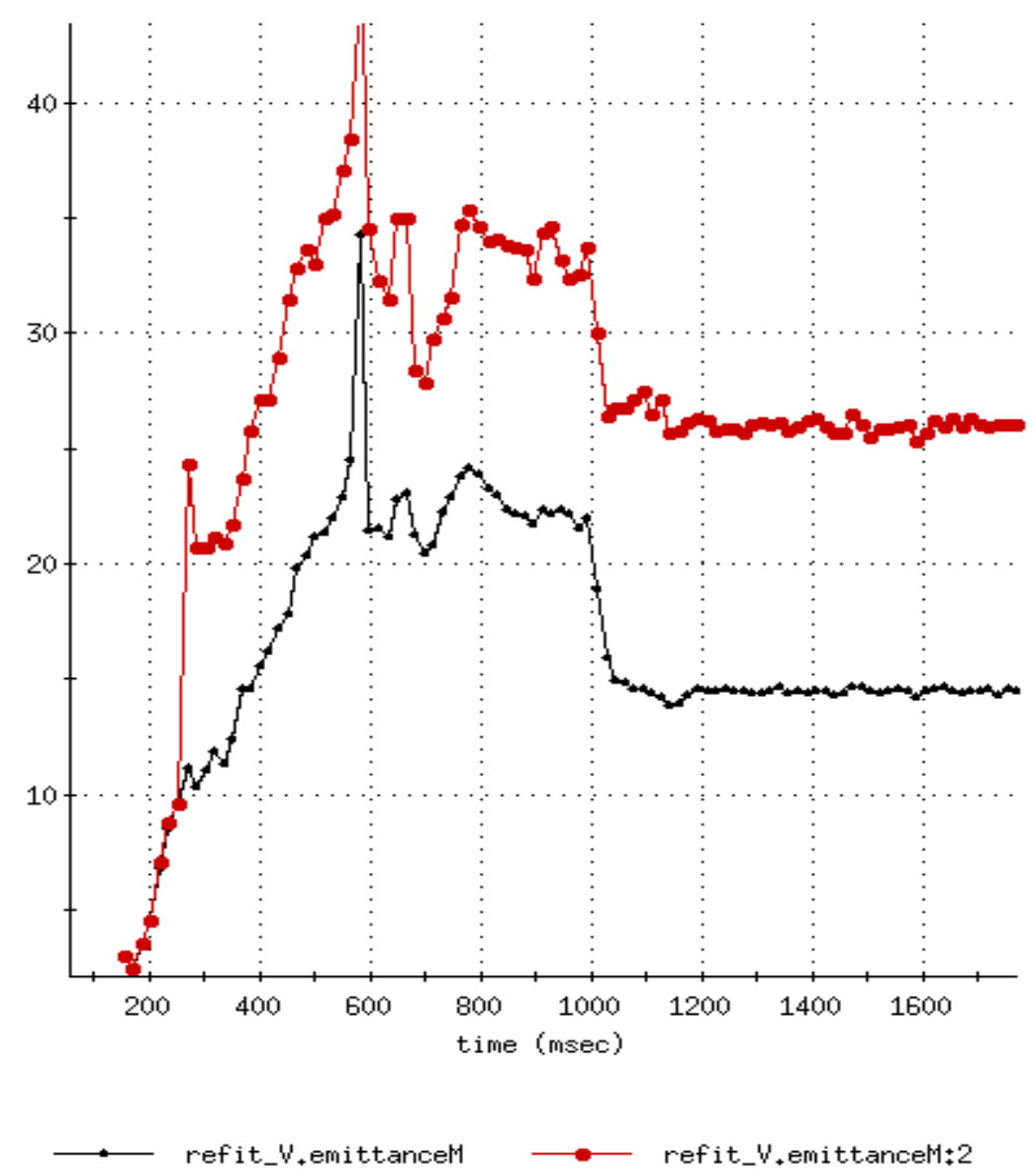

Figure 27: AGS IPM with (red) and without (black) vertical emittance blowup near 0+ (At0+262 ms). The y-axis is $\varepsilon_{\mathrm{y}}$ in $\pi \mathrm{mm} \mathrm{mrad}$; the $\mathrm{x}$-axis is time from At0 in $\mathrm{ms}$. The RF is turned off at At0+1000 ms.

When it was observed that the blowup sometimes occurs and sometimes doesn't for the same size oscillation, and that the amount of blowup was the same regardless of the size of the oscillation, it wasn't appreciated that it might be a clue as to what the source of the blowup is. So, it wasn't investigated any further and I am only aware of this one observation which could very well be just a red herring. Regardless, I believe instabilities that don't change the location of the center of mass are possible in the AGS as they were seen during high intensity proton operation with a single bunch. ${ }^{37}$ It might be that the A3 PUE which is wired into the mux system and was available this run would have the capability to show it.

\footnotetext{
37 High Intensity Beam Operation of the Brookhaven AGS, T. Roser in Workshop on Instabilities of High Intensity Hadron Beams in Rings. BNL, 6/28-7/1/99. Evidence of a Head-Tail instability with a single bunch in the AGS is shown here.
} 


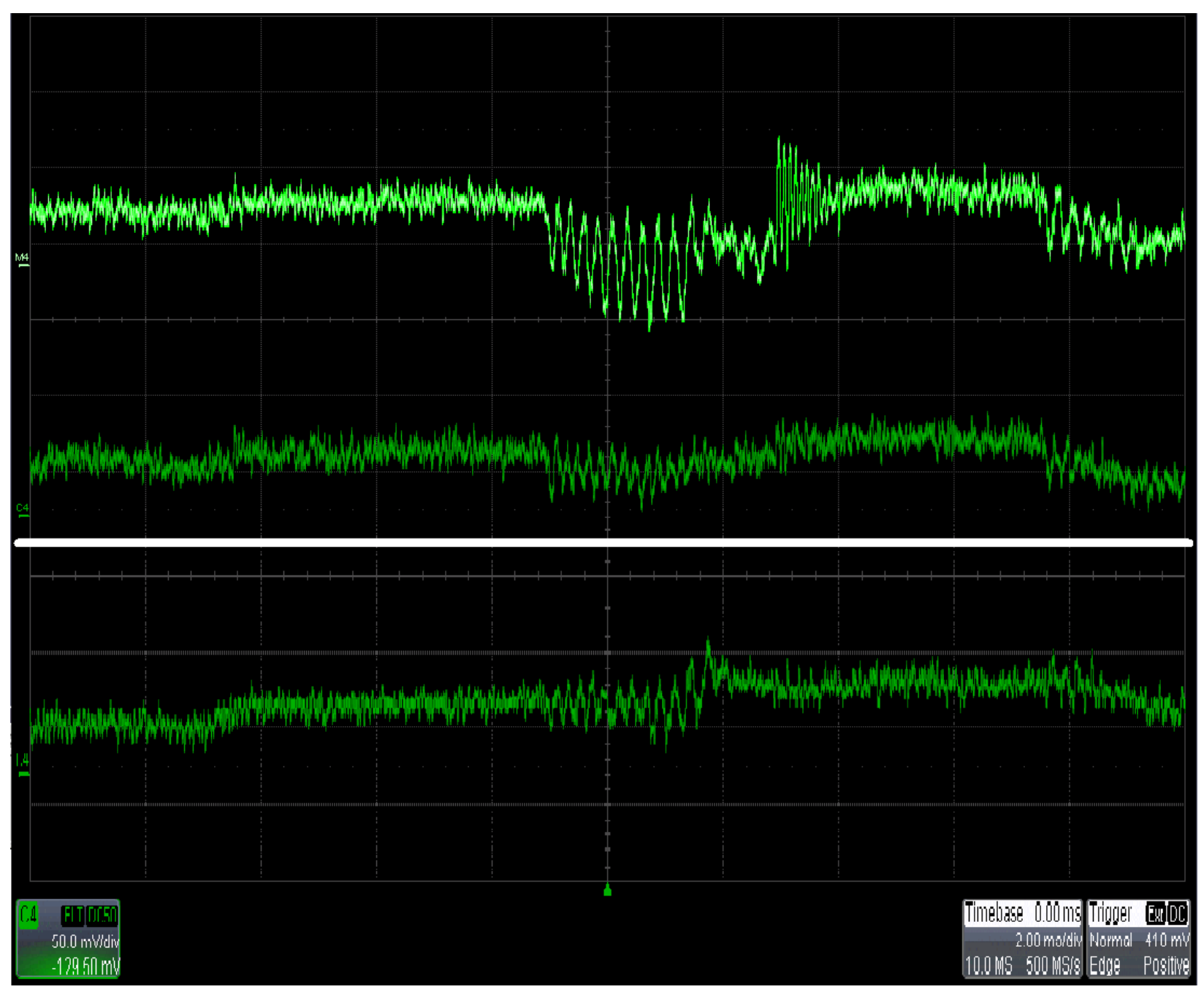

Figure 28: The $\mathrm{CO} 8$ vertical BPM with blowup occurring (top), with it not occurring (middle), and with it occurring (bottom). The trigger is at $\mathbf{2 6 2} \mathrm{ms}$ and sweep speed is $\mathbf{2} \mathrm{ms} / \mathrm{div}$. This is a composite of $\mathbf{2}$ scope dumps, but they both have the same trigger, sweep speed, and horizontal and vertical scales.

Regardless of whether or not this blowup is related to such an instability the question remains as to why it wasn't observed earlier in the run. It was first noticed not long after the problems with deteriorating polarization occurred (Mar 27).

During the latter part of the run, more or less from early April onward, $\mathrm{Q}_{\mathrm{v}}$ around 0+ was typically higher than in the previous part (maybe 4.986 to 4.987 instead of 4.985 to 4.986 ). This change was mostly in response to deteriorating polarization. ${ }^{38}$

\section{The AGS Main Magnet Power Supply Ripple Problem}

During much of the early part of the run (Jan 29 - Mar 18) the AGS was plagued by intermittent and sometimes large longitudinal quadrupole oscillations as observed on the wall

\footnotetext{
${ }^{38}$ See Booster AGS PP elog May 132043 entry for example (where it is measured to be 8.9865).
} 
current monitor. These were correlated with $360 \mathrm{~Hz}$ oscillations on the RF bunch-to-bucket phase signal. Figure 29 shows the oscillations on the wall current monitor and the bunch-tobucket phase signal in a particularly bad state. ${ }^{39}$ Also shown is the station I main magnet power supply voltage.

The severity of these oscillations varied greatly and it is hard to quantify how often the beam quality was affected, but the bad state was far from unusual. When in the bad state there was some deterioration in both longitudinal and transverse emittance. There was also beam loss associated with it.

There was no obvious change in the station I voltage between the good and bad states and its voltage ripple is 720 not $360 \mathrm{~Hz}$. After much investigation by the RF group it turned out that the Station II voltage was changing and when the bunch-to-bucket phase oscillations were in the bad state, the station II voltage had a large $360 \mathrm{~Hz}$ component. ${ }^{40}$ The voltage (or more accurately the $\mathrm{dB} / \mathrm{dt}$ ) that the beam sees is the sum of these 2 stations and they are phased so that the ripples on both stations nearly cancel. But when in the bad state there was a sizable $360 \mathrm{~Hz}$ component on the sum of the station voltages. Once the problem with station II was isolated it was quickly fixed by the power supply group and this quadrupole oscillation problem did not happen again.

\section{The $36+Q_{v}=G \gamma$ Harmonic Bump}

As part of the standard polarized proton setup there is a 'bump' in the vertical $\sin 9 \theta$ component of the AGS closed orbit in the vicinity of the 36+ intrinsic resonance. This is there to aid in maintaining polarization. Its optimal amplitude was thought to be about $+4 \mathrm{~mm}$. It is difficult to maintain as its amplitude drifts and requires frequent attention. Its presence can also result in vertical emittance growth since tune jumps occur near or during it.

It was noticed on May 5 that removing this bump has little or no effect on polarization. ${ }^{41}$ A later bump amplitude scan showed some amplitude dependence though not as much as expected. ${ }^{42}$ This resonance occurs very close to the end of the magnet ramp where the field starts to 'rollover' onto the flattop and the strength of the resonance is related to the ramp rate there. The studies that determined that the bump aided in maintaining polarization were performed with a magnet cycle that has a slower $\mathrm{dB} / \mathrm{dt}$ during this period than the one that's been used since Run 13. Given the difficulty in maintaining this bump and its sometimes negative effect on vertical emittance perhaps further study of the need for it is in order.

\footnotetext{
${ }^{39}$ Booster AGS PP elog Feb 261252 entry

40 Booster AGS PP elog of Mar 171817 and other entries.

41 Booster-AGS PP elog of May5 entries from 1825 to 1905

42 Booster AGS PP elog of May 7, May 81445 entry, V. Schoefer
} 


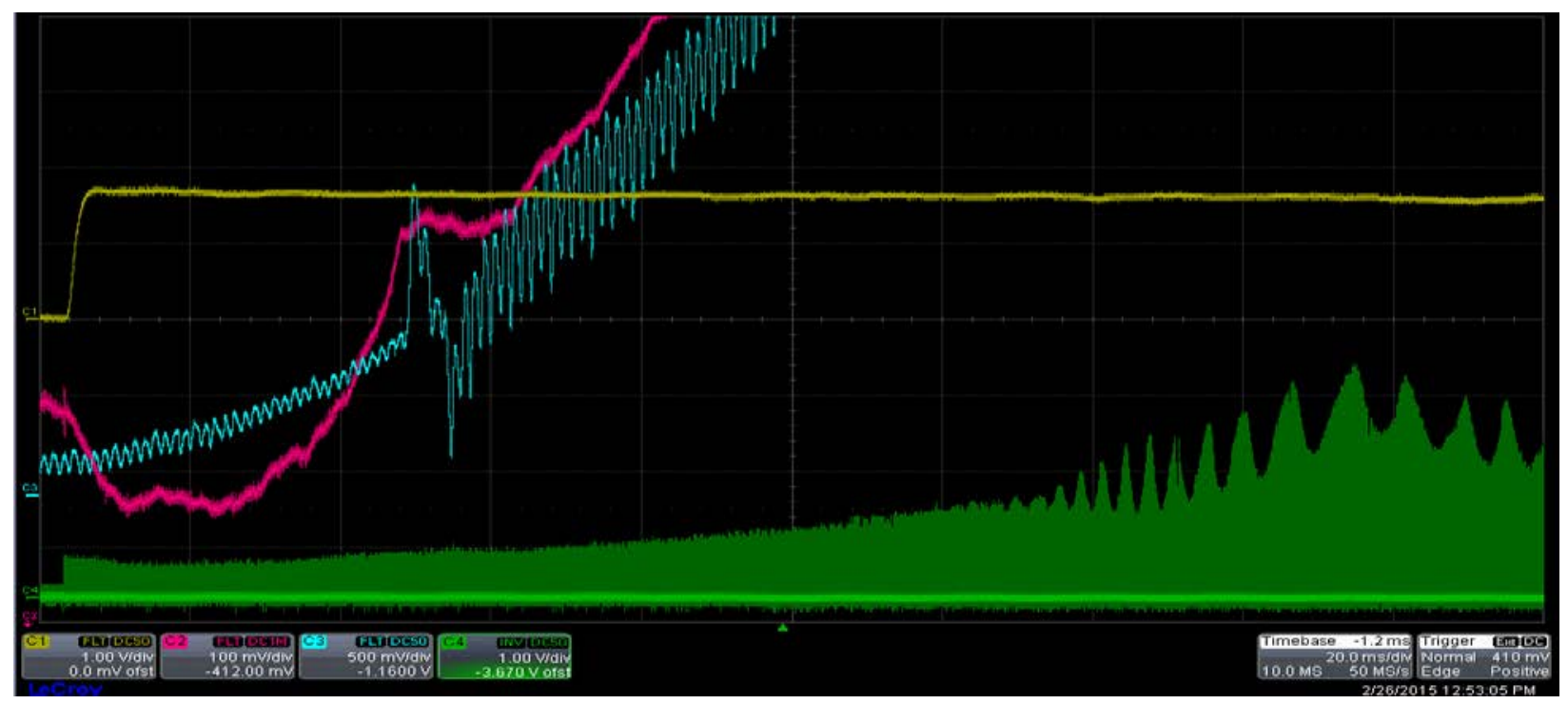

Thu Feb 26 12:52:02 2015, cycle 1424973122

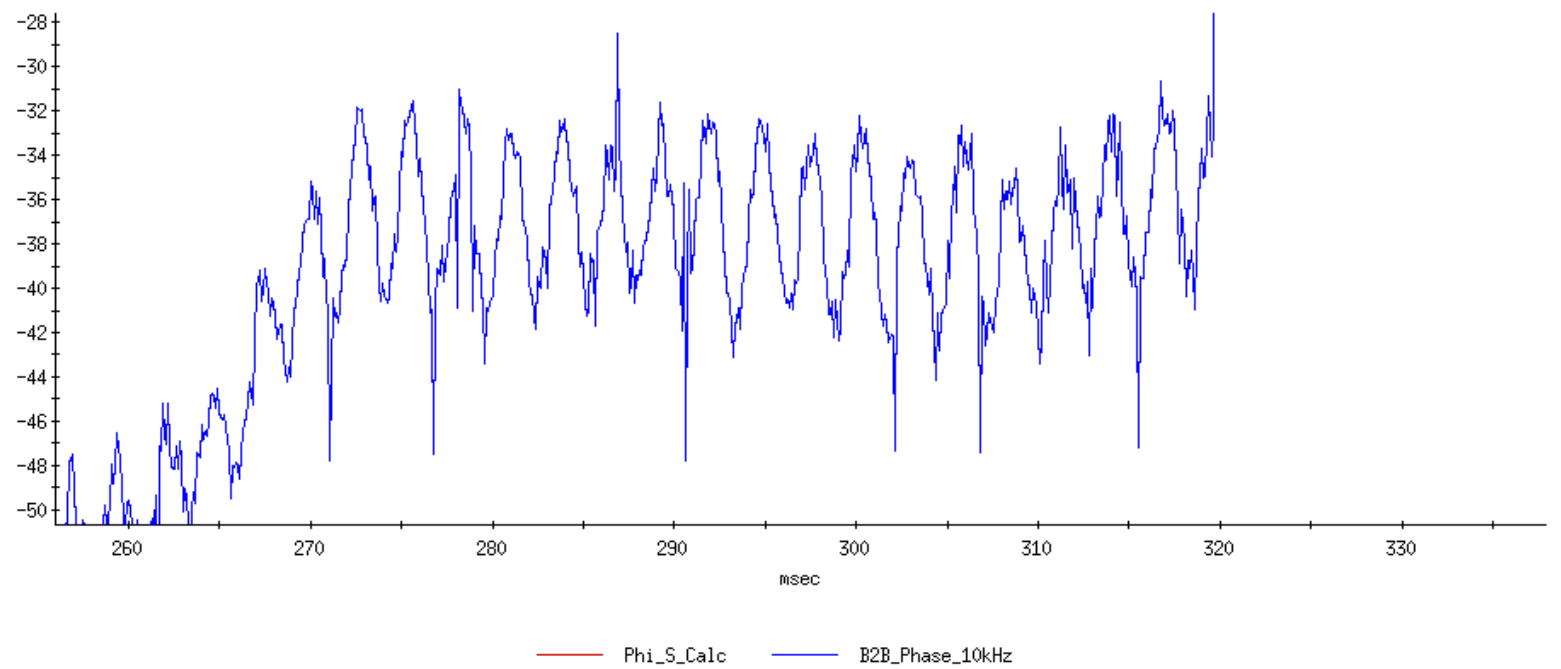

Figure 29: At the top is the AGS Wall Current Monitor (green), the AGS normalized current transformer (yellow), the RF Voltage (red), and the AGS Main Magnet Power Supply Station I voltage (blue). The trigger is At0+240 ms and the sweep speed is $20 \mathrm{~ms} /$ div. On the bottom is the bunch-to-bucket phase where the $x$-axis is time in $\mathrm{ms}$ from At0 and the y-axis is in degrees of the RF wave.

\section{Summary}

This run is notable for the significantly higher polarized proton Linac intensity as compared to previous RHIC runs (8-9e11 vs. 4-5e11) and the attempts to make use of that higher intensity in the presence of what appear to be significant space charge effects in the Booster. In the same vein, the RHIC intensities were higher than in previous runs, and so intensity dependent effects in the AGS may also be playing a more significant role.

In the Booster, a $2^{\text {nd }} \mathrm{RF}$ harmonic was used to flatten out the bunch and thereby reduce the peak charge density in hopes of reducing the effects of space charge (Section 1). Figure 30 
shows BtA and AGS emittances in Runs 13 and 15 as a function of AGS late. ${ }^{43}$ Both the BtA multiwire and the AGS IPM data suggest that the emittance was smaller this year than in Run 13. This doesn't mean that this is solely, or even mainly, due to the 2nd harmonic. First of all, there is more input current and the Booster setup is not entirely dominated by space charge at these intensities. Also, as discussed in section 3, the early vertical tune can be raised and third integer stopbands corrected to mitigate the effects of space charge.

There does however seem to be some improvement in the beam's brightness from the $2^{\text {nd }}$ harmonic. Much of this has to do with a reduction in intensity dependent losses through the early part of the cycle when the $2^{\text {nd }}$ harmonic is used (the late intensity without scraping is about $10 \%$ higher with the $\mathrm{h}=2$ on at an input intensity of $8 \mathrm{e} 11$ ). If any, the effect of the $2^{\text {nd }}$ harmonic on the emittance itself is subtle, though it appears that it may have an effect on the vertical. The vertical profile in BtA is not Gaussian. It has large tails and relatively small core. As the input intensity is increased the core widens until eventually the profile becomes Gaussian. The $2^{\text {nd }}$ harmonic seems to make this transition occur at a higher input intensity (see figure 5).

The Booster uses a half integer injection scheme (Section 4) to reduce the effect of scattering on the foil. There is a concern that it may however cause emittance blowup because of the proximity to the half integer resonances. It appears though that at this run's nominal input intensity (about 9e11) it is still preferable to a normal injection scheme as that scheme was tried and the overall emittance was significantly larger (see Figure 12 and the related discussion). It was observed though that raising the vertical injection tune slightly as the input intensity is increased does improve the BtA emittance, so there is some effect of the half integer stopband but it seems that it can be compensated for, at least to some extent. In addition, immediately after injection the tunes are rapidly shifted away from the half integer before the RF voltage is raised (i.e., before there is significant bunching) to further reduce any effect.

A magnet cycle with a faster early ramp was used for most of the run to raise the energy faster since space charge effects are generally stronger at lower energies (Section 2). However, any improvement from this change was not obvious.

Both horizontal and vertical scraping were used throughout the run. It was found that a ratio of horizontal to vertical scraping of about 2:3 seems to provide the smallest AGS flattop emittance (Section 5). It was also noticed that increasing the amount of horizontal scraping for a fixed AGS late intensity (which was held fixed by reducing the vertical scraping) could reduce the vertical AGS flattop emittance but not the horizontal.

\footnotetext{
${ }^{43}$ The data from Run 13 is taken from the 2013 Booster-AGS PP elog. Unfortunately there is not much 'refit' data in the log and there is no refit data available before the beginning of April. All the IPM data from Run 15 is refit data. MW006 data is used exclusively for the Run 13 BtA data, and the Run 15 data is the same as that used for Figure 16 (which uses both MW006 and MW060).
} 


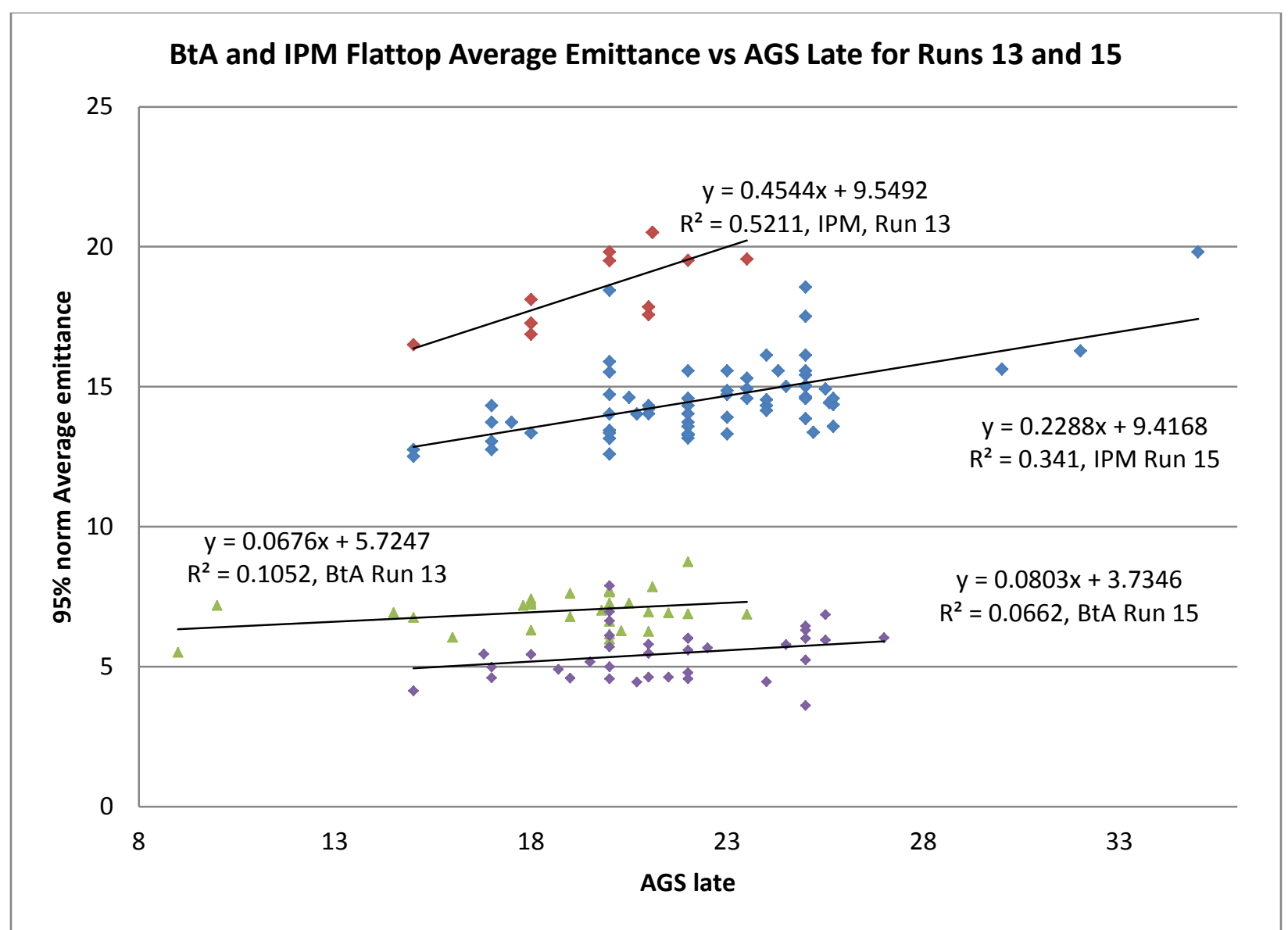

Figure 30: The red and blue points are AGS IPM flattop 'refit' data with RF off from Runs 13 and 15, respectively. The green and purple points are BtA multiwire data from Runs 13 and 15 respectively. Linear fits to the 4 sets of data are included. Average Emittance is in $\pi \mathrm{mm}$ mrad and AGS late is $\times 10^{10}$.

The effect of the tune path during the part of the cycle before 0+ on the AGS flattop emittance was also discussed (Section 6). Generally speaking, the smallest flattop emittance occurs when the tunes are very close to each other and sometimes crossing, especially between 180 and $220 \mathrm{~ms}$ after At0 (see Figures 17 and 18). It is the vertical emittance that gets larger if these tunes are kept far from each other. Given the difficulty in raising $Q_{h}$ past a certain value this means that $\mathrm{Q}_{\mathrm{v}}$ is kept well below the spin tune gap, but no adverse effects of this on polarization have been apparent. This is not a new phenomenon; it has been the case in previous runs.

Given the extended periods of apparently degraded polarization this run, the AGS polarization measurements associated with RHIC fills for Run 15 and Run 13 were compared (Section 7). There was not much data for Run 13 for the AGS late intensities that were often used for RHIC fills during Run 15. Also, the periods of 'degraded' polarization in Run 15 all occurred at relatively high fill intensities (>2e11). But it seems that overall the polarization for Run 15 may have been slightly higher than in Run 13 (see Figure 19). 
The polarization's intensity dependence in the 'high' polarization state was about the same as in Run 13, but the polarization in the 'high' state was on average 2-3\% higher than in Run 13 (see Figure 20). In the 'low', or 'degraded' state the intensity dependence was stronger, and for intensities above 2e11 or so it was lower than in Run 13 (Figure 20).

Next the Run 13, Run 15 'high’ state, and Run 15 'low’ state data was compared but only for AGS late >2e11. The Run 13 data had the steepest intensity dependence, followed by the Run 15 'low' state, and then the Run 15 'high' state. Curiously, the Run 15 'high' state for intensities >2e11 showed no obvious intensity dependence. In that sense the 'high' state seems to be more different from Run 13 than the 'low' state. Also, the linear fit of polarization vs. AGS late for the Run 15 'low' state data gave a higher polarization than the Run 13 data for late intensities above 2.3e11 or so (see Figure 21).

A study on May 8 was performed that indicated that bunches that are wider and less peaked on the first turn in the AGS are correlated with several percent higher polarization and that this effect is not intensity dependent (Section 8). Subsequently, there was a deliberate attempt to make the bunches wider coming out of the Booster. Regardless, there was no apparent improvement in the overall polarization after this approach was adopted. It is not clear how much of an effect this had on the final bunch length, but it is unlikely that it increased it by no more than $10 \%$.

A change to the early flattop tunes was made on Apr 2 (Section 9). This change seemed to make the horizontal IPM flattop emittance about $1 \pi \mathrm{mm}$ mrad larger and the vertical about $2.5 \pi \mathrm{mm}$ mrad smaller. However, despite this change it's not obvious that the overall emittance $\varepsilon_{\text {avg }}$ was smaller subsequent to that (see Figure 26). It appeared that $\varepsilon_{\mathrm{h}}$, as measured by the IPM, increased by about $2 \pi \mathrm{mm}$ mrad during the latter part of the run (Figure 26). But that measurement is very sensitive to $\Delta \mathrm{p} / \mathrm{p}$, and the changes measured in flattop bunch length seem to be enough to account for it.

In early April vertical emittance blowup around 0+ was noticed and lowering $\mathrm{Q}_{\mathrm{v}}$ there could stop it from happening (Section 10). It was thought at first that it was associated with vertical coherence apparent on a BPM and caused by the tune jump firing just before 0+; but later it was noticed that it could still occur even when the amount of that coherence was essentially the same as in the case when it didn't occur (Figures 27 and 28). There is however only this one observation of which I'm aware. But it raises the question of whether there are types of instabilities occurring that our usual diagnostics are insensitive to.

For a good part of the early part of the run (Jan 29-Mar18) there were intermittent but not uncommon periods of large longitudinal bunch shape oscillations during acceleration (Section 11). Eventually these were found to be correlated with changes in the voltage ripple on station II of the AGS main magnet power supply and then the problem was quickly corrected. 
It was noticed during early May that the amplitude of the vertical sin $9 \theta$ harmonic bump used to maintain polarization crossing 36+ did not have as strong of an effect as it once did (Section 12). This may be due to the faster ramp rate in the vicinity of 36+ that the AGS magnet cycle has now. The cycle was changed early in Run 13, and the effect of the sin $9 \theta$ bump had perhaps not been studied in much detail since then. 